\title{
Small knot complements, exceptional surgeries and hidden symmetries
}

\author{
NeIL R HofFMAN
}

\begin{abstract}
This paper provides two obstructions to small knot complements in $S^{3}$ admitting hidden symmetries. The first obstruction is being cyclically commensurable with another knot complement. This result provides a partial answer to a conjecture of Boileau, Boyer, Cebanu and Walsh. We also provide a second obstruction to admitting hidden symmetries in the case where a small knot complement covers a manifold admitting some symmetry and at least two exceptional surgeries.
\end{abstract}

57M12, 57M25; 57M10

\section{Introduction}

Two finite-volume, orientable, hyperbolic 3-orbifolds are commensurable if they share a common finite-sheeted cover. Using Mostow-Prasad rigidity (see Mostow [16] and Prasad [19]), we may restate this definition in terms of Kleinian groups with finite covolume. Namely, $\Gamma_{1}$ and $\Gamma_{2}$ are commensurable if there exists $g \in \operatorname{PSL}(2, \mathbb{C})$ such that $\Gamma_{1}$ and $g \Gamma_{2} g^{-1}$ share a common finite-index subgroup. Commensurability defines an equivalence relation both on the set of hyperbolic 3-orbifolds and on their associated Kleinian groups. One way to distinguish these equivalence classes, called commensurability classes, is to identify elements that are rare in a given commensurability class. Conjecturally, there are at most three hyperbolic knot complements in a given commensurability class (see Reid and Walsh [22, Conjecture 5.2]). Both here and throughout the paper, we will use the term knot complement to refer to a space homeomorphic to $S^{3}-K$, where $K$ is a smoothly embedded knot in $S^{3}$.

In [5], Boileau, Boyer, Cebanu and Walsh show the conjecture holds if the knot complements do not admit hidden symmetries, ie the knot complements do not cover an orbifold with a rigid cusp (see Section 2). Furthermore, two commensurable knot complements either admit hidden symmetries or are cyclically commensurable, ie they both cyclically cover the same orbifold (see [5, Theorem 1.4]). For a small knot complement, ie the knot complement does not contain a closed, embedded, essential surface, Theorem 1.1 below shows these are mutually exclusive, which is a partial solution to [5, Conjecture 4.14]. 
Theorem 1.1 Let $S^{3}-K$ be a small hyperbolic knot complement. If $S^{3}-K$ admits hidden symmetries, it is not cyclically commensurable with another knot complement.

We now provide an outline of the proof of Theorem 1.1. First, we ignore the figure- 8 knot complement as it is the only knot complement in its commensurability class (see Reid [21]). The key idea of the proof is to examine a covering $p: Q_{T} \rightarrow Q$, where both $Q_{T}$ and $Q$ are orbifolds covered by $S^{3}-K, Q_{T}$ has a torus cusp and $Q_{T}$ admits (at least) two finite cyclic fillings, and $Q$ is a rigid cusped orbifold. As noted above, the existence of such a $Q_{T}$ is established in [5], $Q$ is established by assumption, and the covering is established by Margulis [11]. Furthermore, by [5], $Q_{T}$ both is covered by (at least) two knot complements and is the complement of a knot in the orbifold equivalent of a lens space. Also by [5], the singular set of such an orbifold is either empty or comprised of a single circle. In Section 2.3, we establish a tool for understanding the basic structure of the singular set of $Q$, which we refer to as the cusp-killing homomorphism. The power of this tool can be seen in Lemma 4.3, which summarizes the combinatorial arguments of Section 4 regarding the singular sets of $Q_{T}$ and $Q$ and provides a lower bound on $\operatorname{deg}(p)$. However, since $S^{3}-K$ is small, we may use Bass [4] to assume that $\pi_{1}\left(S^{3}-K\right)$ admits integral traces (see Section 2). Next, using Lemmas 3.1 and 3.2, we exhibit a specific representation of $\pi_{1}\left(S^{3}-K\right)$ where the image of the representation includes an upper triangular meridian having a unit in the off-diagonal entry. By Maclachlan and Reid [10, Lemma 5.2.4], the smallness of $S^{3}-K$ implies the existence of an integral representation of $\pi_{1}^{\text {orb }}(Q)$ into $\operatorname{PSL}(2, \mathbb{C})$. Hence meridians of this form are rare. By analysis of the covering restricted to the cusp, we can establish an upper bound on $\operatorname{deg}(p)$, since $Q_{T}$ is covered by more than one knot complement and therefore $\pi_{1}^{\text {orb }}\left(Q_{T}\right)$ must contain two distinct upper triangular meridians. Finally, the upper and lower bounds do not agree and this contradiction establishes the theorem.

Lemmas 3.1 and 3.2 are also key tools for showing that a small knot complement covering an orbifold with a rigid cusp cannot cover a manifold that admits exceptional surgeries and some symmetry. Typically, obstructions to covering rigid cusped orbifolds arise from the computation of arithmetic invariants, eg the invariant trace field (see Section 2). The following theorem provides an obstruction to covering a rigid cusped orbifold by exploiting information of a more topological and geometric nature.

Theorem 1.2 Let $M$ be a manifold covered by a small hyperbolic knot complement $S^{3}-K$ that is not the figure-8 knot complement.

(1) If $M$ admits two exceptional surgeries, then $M$ does not cover an orbifold with a $S^{2}(2,4,4)$ or $S^{2}(3,3,3)$ cusp.

(2) If $M$ admits two exceptional surgeries and a nontrivial symmetry, then $M$ does not cover an orbifold with a $S^{2}(2,3,6)$ cusp. 
As noted above, the common thread in the proofs of Theorems 1.1 and 1.2 is the use of Lemmas 3.1 and 3.2. In the proof of Theorem 1.2, we also appeal to the six theorem of Agol [2] and Lackenby [9]. To incorporate these two ideas, we say that $p: M \rightarrow Q$, where $M$ is a manifold as in Theorem 1.2 and $Q$ is a rigid cusped orbifold. Instead of establishing an upper bound on $\operatorname{deg}(p)$, Lemmas 3.1 and 3.2 together with the six theorem combine to show that $M$ has two short curves in its cusp. These short curves place an upper bound on the cusp volume of $M$ (see Section 5.1). The arguments in Section 5 also extend the combinational investigation of the singular set of $Q$ used for the proof of Theorem 1.1. Together with the symmetry assumption, this more refined analysis establishes a lower bound on $\operatorname{deg}(p)$, which in turn yields an upper bound on the cusp volume of $Q$. The upper bound depends on the cusp type of $Q$ (see Lemma 5.5). In fact, if $Q$ has an $S^{2}(2,4,4), S^{2}(3,3,3)$ or $S^{2}(2,3,6)$ cusp, then the bounds for the cusp volume of $Q$ are $3 / 24, \sqrt{3} / 4$ and $\sqrt{3} / 8$ respectively (assuming we use the symmetry of $M$ in the $S^{2}(2,3,6)$ case). Such bounds line up almost perfectly with Adams' classification of orbifolds with small cusp volume, which is restated for the sake of completeness as Theorem 2.1 (also see Adams [1]). Therefore, the remainder of the proof comes down to observing properties about these orbifolds. Some of this work is already a part of the literature as summarized in Proposition 2.2 (see [1] and Neumann and Reid [18] for further background) and Neumann and Reid [17, Section 9]. The final cases are addressed in Lemmas 5.2 and 5.4.

This paper is organized as follows. In Section 2, we provide the necessary background including a description of many of the smallest volume cusped orbifolds. We then establish two lemmas that together characterize the representations of the fundamental groups of small knot complements in $\operatorname{PSL}(2, \mathbb{C})$ in Section 3. In Section 4, we exhibit some combinatorial properties of a rigid cusped orbifold covered by a knot complement and the singular set of such an orbifold, followed by a proof of Theorem 1.1. And in the following section, we obstruct certain orbifolds from being covered by knot complements followed by a proof of Theorem 1.2. In Section 6, we provide some final remarks along with a theorem that makes our results from [7] more explicit.

Acknowledgements This work started under the direction of my $\mathrm{PhD}$ advisor Alan Reid, who patiently and thoughtfully provided feedback on this work at every stage of development. He deserves many thanks for this. Some of the final work was done while I was supported by Boston College. Also, I would like to thank Colin Adams, Ken Baker, Jason Deblois, Cameron Gordon, Brandy Guntel, Keenan Kidwell, John Luecke, Matthew Stover and Genevieve Walsh for a number of helpful conversations. Finally, I would like to thank the referee for many thoughtful suggestions. 


\section{Background}

We begin by establishing some notation. First, we consider all groups as subgroups of PSL $(2, \mathbb{C})$ with finite covolume unless explicitly stated otherwise. Therefore, we consider all manifolds and orbifolds to be both hyperbolic and orientable unless explicitly stated otherwise. We denote $\Gamma_{Q}=\pi_{1}^{\text {orb }}(Q)$. Hence we may say $Q=\mathbb{H}^{3} / \Gamma_{Q}$. In the case of knot complements, we say that $\Gamma_{K}=\pi_{1}\left(S^{3}-K\right)$. Furthermore, it will prove convenient to discuss the subgroup that fixes $\infty$. We call such a subgroup the peripheral subgroup of $\Gamma_{Q}$ (or $\Gamma_{K}$ ) and denote it by $P_{Q}$ (or $P_{K}$ ).

A distinct advantage of considering groups as discrete subgroups of $\operatorname{PSL}(2, \mathbb{C})$ is that they carry a significant amount of number theoretic information. In order to fully utilize this data, we observe the following standard notation. First, we denote the set of algebraic integers in $\mathbb{C}$ by $\mathbb{A}$. If $L$ is a number field, we will use $\mathcal{O}_{L}$ to denote the ring of integers in $L$ and $\alpha \mathcal{O}_{L}$ to denote the principal ideal generated by $\alpha \in \mathcal{O}_{L}$. If $\mathcal{I}$ is a nonprincipal ideal in $\mathcal{O}_{L}$, we will denote it by $\langle\alpha, \beta\rangle$, where $\mathcal{I}$ is generated by the $\mathcal{O}_{L}$ linear combinations of $\alpha$ and $\beta$.

For a group $\Gamma, g \in \Gamma$ is a two element coset of the form

$$
\left\{\left(\begin{array}{ll}
a & b \\
c & d
\end{array}\right),\left(\begin{array}{ll}
-a & -b \\
-c & -d
\end{array}\right)\right\}
$$

Later, we will abuse notation and just refer to an element by one of the above matrices. We say the trace of $g$ or $\operatorname{tr}(g)=a+d$. Although this definition is only well-defined up to sign, it will be sufficient for our purposes. For example, if for all $g \in \Gamma, \operatorname{tr}(g) \in \mathbb{A}$, we say $\Gamma$ has integral traces. Otherwise, $\Gamma$ has nonintegral traces. In addition, if $\Gamma$ has integral traces, then $\Gamma$ admits a representation into $\operatorname{PSL}(2, \mathbb{A})$, a fact exploited in Lemma 3.1.

Next, we define the invariant trace field of $\Gamma$ or $k \Gamma$ to be $\mathbb{Q}\left(\left\{\operatorname{tr}\left(g^{2}\right) \mid g \in \Gamma\right\}\right)$. In [20], Reid showed that this is an invariant of the commensurability class of $\Gamma$. We also a define the trace field of $\Gamma$ to be $\mathbb{Q}(\{\operatorname{tr}(g) \mid g \in \Gamma\})$.

A cusped 3-orbifold $Q=\mathbb{H}^{3} / \Gamma$ (or $\Gamma$ ) is arithmetic if $\Gamma$ is commensurable with $\operatorname{PSL}\left(2, \mathbb{O}_{d}\right)$, where $\mathbb{O}_{d}$ is the ring of integers in $\mathbb{Q}(\sqrt{-d})$ and $d$ is a positive squarefree integer. Otherwise, $Q$ (or $\Gamma$ ) is nonarithmetic.

\subsection{Rigid cusps}

The cusps of an orientable 3-orbifold are one of five types (see Walsh [25, Section 3] for background). The nonrigid Euclidean 2-orbifolds are the torus and $S^{2}(2,2,2,2)$. The rigid orbifolds are $S^{2}(2,4,4), S^{2}(2,3,6)$ and $S^{2}(3,3,3)$. 
It is worth mentioning that the definition of hidden symmetries in the introduction is slightly atypical. However, the argument below shows it equivalent to the standard definition. First, we denote by $\operatorname{Comm}(\Gamma)$ the (orientable) commensurator of $\Gamma$ in $\operatorname{PSL}(2, \mathbb{C})$, or

$$
\operatorname{Comm}(\Gamma)=\left\{g \in \operatorname{PSL}(2, \mathbb{C}) \mid \begin{array}{r}
{\left[g \Gamma g^{-1}: g \Gamma g^{-1} \cap \Gamma\right]<\infty} \\
{\left[\Gamma: g \Gamma g^{-1} \cap \Gamma\right]<\infty}
\end{array}\right\} .
$$

Traditionally, a group $\Gamma$ admits hidden symmetries if $[\operatorname{Comm}(\Gamma): N(\Gamma)]>1$, where $N(\Gamma)$ is the normalizer of $\Gamma$ in $\operatorname{PSL}(2, \mathbb{C})$. Also, we say $Q$ admits hidden symmetries if $\Gamma_{Q}$ admits hidden symmetries. In the case where $\Gamma$ is nonarithmetic, $\operatorname{Comm}(\Gamma)$ is discrete in $\operatorname{PSL}(2, \mathbb{C})$ and we can define $\mathbb{H}^{3} / \operatorname{Comm}(\Gamma)$ as the (orientable) commensurator quotient (see [11]). By construction, for nonarithmetic $\Gamma, \mathbb{H}^{3} / \operatorname{Comm}(\Gamma)$ is covered by all orbifolds in its commensurability class and therefore it is the smallest volume orbifold in the commensurability class of $\mathbb{H}^{3} / \Gamma$. Also, for a nonarithmetic knot complement, having hidden symmetries is equivalent to $\mathbb{H}^{3} / \operatorname{Comm}\left(\Gamma_{K}\right)$ having a rigid cusp by [17, Proposition 9.1]. The only arithmetic knot complement is the figure8 knot complement (see [21]) and the figure- 8 knot complement covers a rigid cusped orbifold while admitting hidden symmetries. Thus the definition is also equivalent for this case.

In arguments that follow, we will need to exploit facts about many of the smallest volume cusped orbifolds, all of which have rigid cusps. This accounting of small volume orbifolds heavily relies on Meyerhoff's result that the densest horoball packing has a cusp density of $\sqrt{3} /\left(2 v_{0}\right)$, where cusp density is defined to be cusp volume divided by total volume and $v_{0} \approx 1.0149416$ is the volume of the regular ideal tetrahedron (see Meyerhoff [13]).

The following theorem summarizes Meyerhoff's result and Adams' classification of small cusp-volume hyperbolic orbifolds (see [1, Theorem 3.2, Corollary 4.1, Theorem 5.2]).

Theorem 2.1 (Adams, 1991) Let $Q$ be a 1-cusped hyperbolic 3-orbifold.

(1) A maximal $S^{2}(2,3,6)$ cusp in $Q$ has volume either $\sqrt{3} / 24, \sqrt{3} / 12,1 / 8$, $(\sqrt{3}(3+\sqrt{5})) / 48, \sqrt{21} / 24$ or at least $\sqrt{3} / 8$.

(2) A maximal $S^{2}(3,3,3)$ cusp in $Q$ has volume either $\sqrt{3} / 12, \sqrt{3} / 6,1 / 4$, $(\sqrt{3}(3+\sqrt{5})) / 24, \sqrt{21} / 12$ or at least $\sqrt{3} / 4$.

(3) A maximal $S^{2}(2,4,4)$ cusp in $Q$ has volume either $1 / 8, \sqrt{2} / 8$, or at least $1 / 4$. 
Adams points out that for each cusp volume explicitly listed in (1), there is a unique orbifold $Q$ with a $S^{2}(2,3,6)$ cusp. Moreover, each of these orbifolds has a unique double cover with a $S^{2}(3,3,3)$ cusp corresponding to an orbifold with cusp volume explicitly listed in (2).

Neumann and Reid provided detailed descriptions of many of the orbifolds corresponding to the cusp volumes in these theorems (see [18]). A number of the orbifolds they describe are arithmetic. Adams also notes the volumes of many of these manifolds in [1]. Adopting the standard notation that $v_{1} \approx .91596244$ is the volume of the ideal tetrahedron in $\mathbb{H}^{3}$ with dihedral angles of $\pi / 2, \pi / 4$ and $\pi / 4$, we can summarize the necessary information from these two papers in the following proposition.

Proposition 2.2 (Adams 1991, Neumann and Reid 1991) Let $Q$ be a 1-cusped hyperbolic 3-orbifold.

(1) If $Q$ has a maximal $S^{2}(2,3,6)$ cusp of volume either $\sqrt{3} / 24, \sqrt{3} / 12$ or $1 / 8$, it is arithmetic. Moreover, these orbifolds have volumes $v_{0} / 12, v_{0} / 6$ and $5 v_{0} / 24$, respectively, and no other orbifold with this cusp type is of lower volume.

(2) If $Q$ has a maximal $S^{2}(3,3,3)$ cusp of volume either $\sqrt{3} / 12, \sqrt{3} / 6$ or $1 / 4$, it is arithmetic. Moreover, these orbifolds have volumes $v_{0} / 6, v_{0} / 3$ and $5 v_{0} / 12$, respectively, and no other orbifold with this cusp type is of lower volume.

(3) If $Q$ has a maximal $S^{2}(2,4,4)$ cusp of volume either $1 / 8$ or $\sqrt{2} / 8$, it is arithmetic. Moreover, these orbifolds have volumes $v_{1} / 6$ and $v_{1} / 4$, respectively, and no other orbifold with this cusp type is of lower volume.

Moreover, the only orbifold with a $S^{2}(2,3,6)$ cusp and cusp volume $\sqrt{3}(3+\sqrt{5}) / 48$ is the tetrahedral orbifold $\mathbb{H}^{3} / \Gamma(5,2,2 ; 2,3,6)$ with volume approximately 0.343003 (see [17, Section 9] and [10, page 144] for background). As noted by Adams, this orbifold has a unique 2 -fold cover with a $S^{2}(3,3,3)$ cusp and cusp volume $\sqrt{3}(3+\sqrt{5}) / 24$. This orbifold is $\mathbb{H}^{3} / \Gamma(5,2,2 ; 3,3,3)$ (see [1]).

We delay discussion of orbifolds of other relevant cusp volumes until Section 5.

\subsection{The isotropy graph and finite subgroups of $\operatorname{SO}(3, \mathbb{R})$}

This subsection draws upon Thurston's definition of a (geometric) 3-orbifold. For further background, we refer the reader to Thurston [24, Chapter 13] and [25].

We define the base space of an orbifold $Q$ to be the underlying topological space. For convenience, we use $|Q|$ to denote the base space of an orbifold $Q$. In dimensions 2 and $3,\left|\mathbb{R}^{2} /\left\langle\gamma \mid \gamma^{n}\right\rangle\right|=\mathbb{R}^{2}$ and $\left|\mathbb{R}^{3} / G\right|=\mathbb{R}^{3}$, where $G$ is a finite subgroup of 
$\mathrm{SO}(3, \mathbb{R})$. Hence the base space of a 2 -orbifold is a surface and the base space of a 3-orbifold is a 3-manifold. Also, if all neighborhoods of $x \in Q$ map to $\mathbb{R}^{2} /\left\langle\gamma \mid \gamma^{n}\right\rangle$ or $\mathbb{R}^{3} / G$, we call $x$ a cone point of $Q$.

An elliptic 2-orbifold is an orientable 2-orbifold that can be covered by $\mathbb{S}^{2}$. The complete list of orientable 2-orbifolds covered by $\mathbb{S}^{2}$ is $\mathbb{S}^{2}, \mathbb{S}^{2}(n, n), \mathbb{S}^{2}(2,2, n)$, $\mathbb{S}^{2}(2,3,3), \mathbb{S}^{2}(2,3,4)$ and $\mathbb{S}^{2}(2,3,5)$.

Taking the cone over each of these orbifolds produces all of the possibilities for $\mathbb{R}^{3} / G$ (see Figure 1). In particular, $G$ is either trivial, finite cyclic, $D_{n}$ (a dihedral group of order $2 n), A_{4}, S_{4}$ or $A_{5}$. Finally, if $x \in \mathbb{H}^{3} / \Gamma$ the isotropy group of $x$ is $G \subset \Gamma$ such $g \in G$ if and only if $g(x)=x$. The set of all points fixed by some element in a fundamental domain for $Q$ is a trivalent graph, which we will refer to as the isotropy graph. We note that this graph need not be connected. However, we may consider an orbifold as a base space together with an embedded isotropy graph.
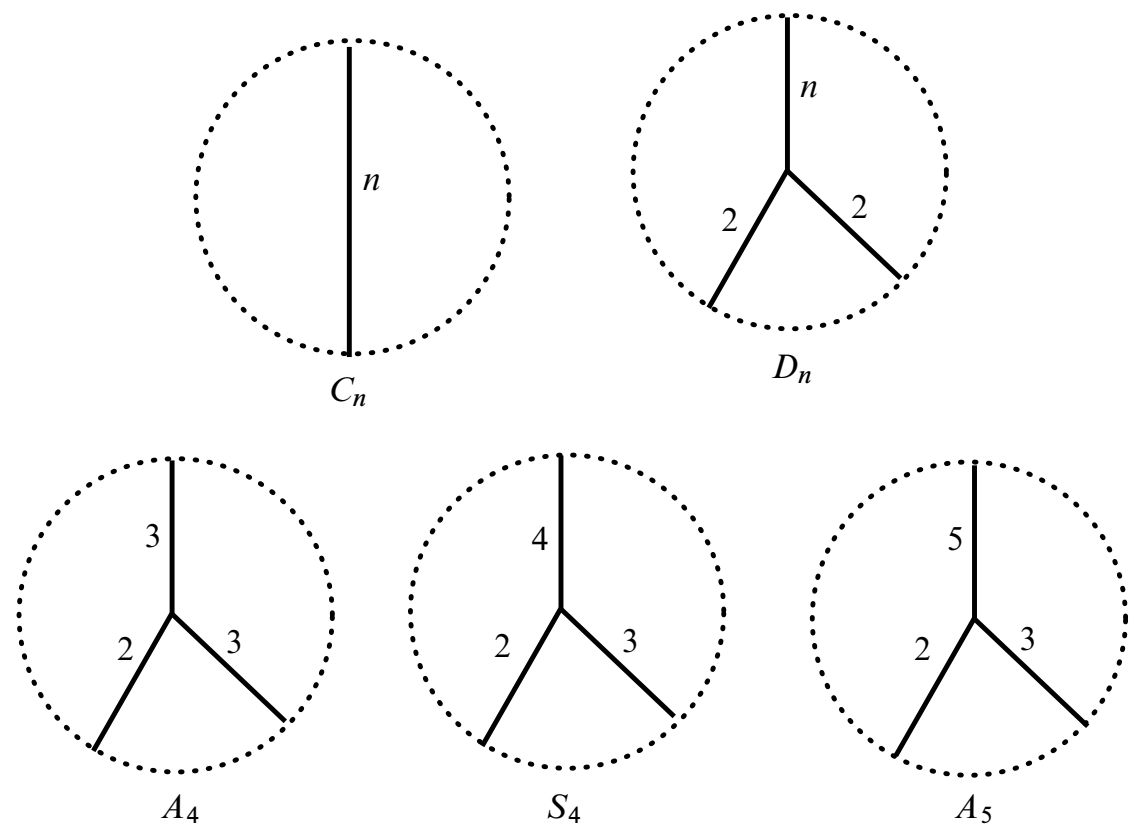

Figure 1: The five types of trivalent points that correspond to finite subgroups of $\operatorname{SO}(3, \mathbb{R})$

The specific embedding of the isotropy graph will be useful in the arguments of this paper. We will pay particular interest to the isometric embeddings of the vertices of the isotropy graph. Using the spherical cosine law (see Roeder [23, Lemma 3] for an application in this context), we can compute the angles between the axes for each 
type of isotropy group in Figure 1. If we consider the three axes fixed by elements of torsion of orders $a, b$ and $c$ where the angles between these axes are $\alpha, \beta, \gamma$ (see in Figure 2), then

$$
\begin{aligned}
& \cos \alpha=\frac{\cos \frac{\pi}{a}+\cos \frac{\pi}{b} \cos \frac{\pi}{c}}{\sin \frac{\pi}{b} \sin \frac{\pi}{c}}, \\
& \cos \beta=\frac{\cos \frac{\pi}{b}+\cos \frac{\pi}{a} \cos \frac{\pi}{c}}{\sin \frac{\pi}{a} \sin \frac{\pi}{c}}, \\
& \cos \gamma=\frac{\cos \frac{\pi}{c}+\cos \frac{\pi}{a} \cos \frac{\pi}{b}}{\sin \frac{\pi}{a} \sin \frac{\pi}{b}} .
\end{aligned}
$$

This table lists some particular values of $(\alpha, \beta, \gamma)$ for given $(a, b, c)$ :

$$
\begin{array}{lc}
(a, b, c) & (\alpha, \beta, \gamma) \\
(2,2, n) & \left(\frac{\pi}{2}, \frac{\pi}{2}, \frac{\pi}{n}\right) \\
(2,3,3) & \left(\cos ^{-1}\left(\frac{1}{3}\right), \cos ^{-1}\left(\frac{1}{\sqrt{3}}\right), \cos ^{-1}\left(\frac{1}{\sqrt{3}}\right)\right) \\
(2,3,4) & \left(\cos ^{-1}\left(\frac{1}{\sqrt{3}}\right), \frac{\pi}{4}, \cos ^{-1}\left(\frac{\sqrt{2}}{\sqrt{3}}\right)\right) \\
(2,3,5) & \left(\cos ^{-1}\left(\frac{\cos \left(\frac{\pi}{5}\right)}{\sqrt{3} \sin \left(\frac{\pi}{5}\right)}\right), \cos ^{-1}\left(\frac{1}{2 \sin \left(\frac{\pi}{5}\right)}\right), \cos ^{-1}\left(\frac{2 \cos \left(\frac{\pi}{5}\right)}{\sqrt{3}}\right)\right)
\end{array}
$$

We note that only the dihedral isotropy groups have the property that there is an axis perpendicular to all other fixed-point axes.

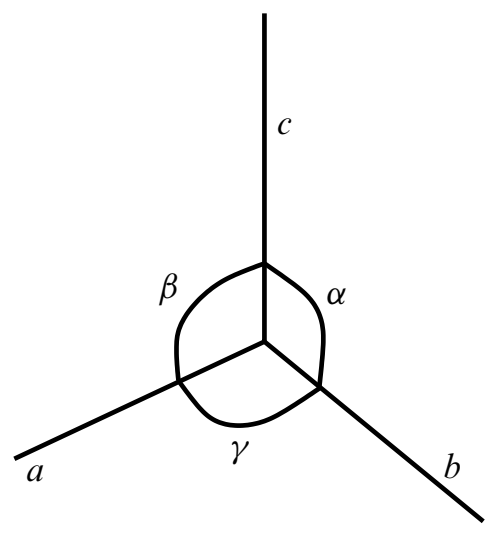

Figure 2: The angles between axes of fixed points in finite subgroups of $\mathrm{SO}(3, \mathbb{R})$ 


\subsection{The cusp-killing homomorphism}

Let $Q=\mathbb{H}^{3} / \Gamma_{Q}$ be a 1-cusped hyperbolic 3-orbifold. Denote by $|\gamma|$ the order of an element in $\Gamma_{Q}$ and denote $R=\left\{\gamma\left|\gamma \in P_{Q},\right| \gamma \mid<\infty\right\}$. We define the cusp-killing homomorphism to be

$$
f: \Gamma_{Q} \rightarrow \Gamma_{Q} /\langle\langle R\rangle\rangle_{\Gamma_{Q}}
$$

We will make use of the following proposition, which was also noticed by M Kapovich.

Proposition 2.3 Let $S^{3}-K$ be a hyperbolic knot complement. Suppose $S^{3}-K$ covers an orientable orbifold $Q$ with a nontorus cusp. Denote the cusp-killing homomorphism by $f$. Then $f\left(\Gamma_{Q}\right)$ is trivial. Furthermore, $|Q| \cong D^{3}$ and each component of the isotropy graph of $Q$ is connected to the cusp.

Proof First note that $\Gamma_{Q}=P_{Q} \cdot \Gamma_{K}$.

Since a meridian $\mu$ of $\Gamma_{K}$ is contained in $P_{Q}$ and $P_{Q}$ is generated by torsion elements on the cusp (we recall Section 2.1), killing these torsion elements kills $\langle\langle\mu\rangle\rangle_{\Gamma_{Q}}$ as well as killing $P_{Q}$. However, $\Gamma_{K}=\langle\langle\mu\rangle\rangle_{\Gamma_{K}}$ and $\langle\langle\mu\rangle\rangle_{\Gamma_{K}} \subset\langle\langle\mu\rangle\rangle_{\Gamma_{Q}}$. Hence the cusp-killing homomorphism kills the whole group $\Gamma_{Q}$.

Thus $|Q|$ is a simply connected space with $S^{2}$ boundary. Therefore, $|Q| \cong D^{3}$ by the solution to the Poincaré conjecture (see Morgan and Tian [15]).

If there were any pieces of the isotropy graph not connected to the cusp, then there would be elements of finite order that are nontrivial under the cusp-killing homomorphism. Hence each component of the isotropy graph is connected to the cusp.

Remark 2.4 We can also interpret the effects of the cusp-killing homomorphism on the isotropy graph of $Q$ when $|Q|$ is simply connected. Viewing the isotopy graph as a weighted graph that generates the fundamental group of $Q$ via the Wirtinger presentation, killing elements of torsion on the cusp corresponds to erasing edges of the graph. For each endpoint $x$ of an erased edge corresponding to an elliptic element $\gamma$, we introduce the relation $\gamma=1$ in the local isotropy group at $x$. If $x$ corresponds to a $S^{2}(2,2,2,2)$ cusp, then the new isotropy group at $x$ is a quotient of the Klein 4 group. If not, then $x$ corresponds to a trivalent vertex of the isotropy graph, say each edge corresponds to torsion elements $\gamma, a$ and $b$. Introducing the relation that $\gamma=1$, to $a b \gamma=1$ yields $a=b^{-1}$. In particular $a$ and $b$ have the same order. Therefore, in the image $f\left(\Gamma_{Q}\right), f(a)=f\left(b^{-1}\right)$ and graphically we can relabel the weights corresponding to $a$ and $b$ with $\operatorname{gcd}(|a|,|b|$ ) (see Figure 3). Relabeling the edges could introduce further reductions to the graph. However, since the isotropy graph has a finite number of vertices and edges and each edge is weighted by a finite integer, this process will terminate in a finite number of steps. 


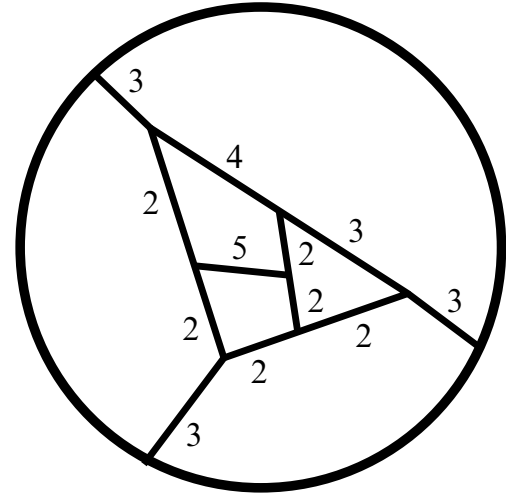

(a) The isotropy graph before reduction

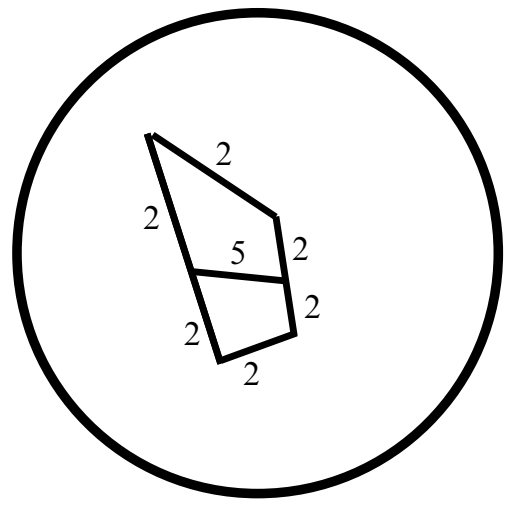

(c) The graph after resolving the degree- 2 vertices

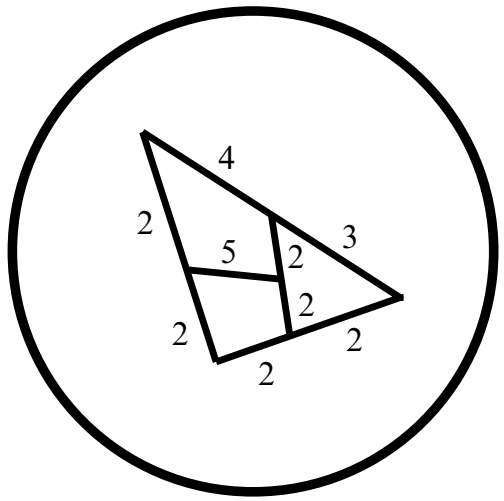

(b) The graph after removing edges corresponding to torsion elements fixing points on the cusp

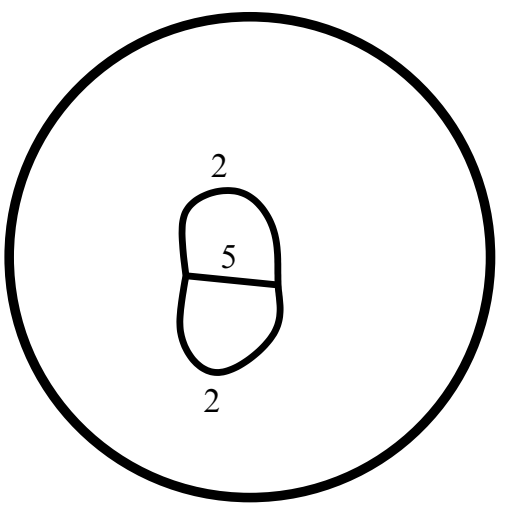

(d) The result of cusp-killing is a graph corresponding to a dihedral group

Figure 3: A step by step graphical interpretation of the cusp-killing homomorphism

\section{Integral representations of knot groups}

In this section, we provide two lemmas about the representations of knot groups into $\operatorname{PSL}(2, \mathbb{A})$. Both main theorems rely on these two lemmas in order to limit the possible covers for $p: S^{3}-K \rightarrow Q$, where $\Gamma_{K}$ admits integral traces (see Section 2) and $Q$ has a rigid cusp.

The key tool from number theory used in the proofs of these lemmas is the Hilbert class field. For further background on the Hilbert class field we refer the reader to Koch [8, Section 10.2]. In the context of this paper, this field is special in two ways. 
First, if $E$ is the Hilbert class field of $L$, then any ideal $\mathcal{I}$ in $\mathcal{O}_{L}$ is principal in $\mathcal{O}_{E}$. The second fact that we exploit is that $E$ is an unramified extension of $L$. By this we mean that for each prime ideal $\mathcal{P}$ of $\mathcal{O}_{L}, \mathcal{P}$ either remains prime or $\mathcal{P}$ splits into the product of distinct prime ideals in $\mathcal{O}_{E}$, that is $\mathcal{P}=\prod_{i=1}^{m} \mathcal{Q}_{i}$, where each $\mathcal{Q}_{i}$ is prime in $\mathcal{O}_{E}$ and $\mathcal{Q}_{i} \neq \mathcal{Q}_{j}$ for all $i \neq j$.

Lemma 3.1 Let $\Gamma_{K}$ be a knot group admitting integral traces. Then for any $Q$ such that $S^{3}-K$ covers $Q, \Gamma_{Q}$ admits a representation into $\operatorname{PSL}(2, \mathbb{A})$ where $\Gamma_{K}$ has an upper-triangular meridian.

Proof Since $\Gamma_{K}$ admits integral traces, $\Gamma_{Q}$ admits integral traces. Therefore, there is an integral representation of $\Gamma_{Q}$ into $\operatorname{PSL}\left(2, \mathcal{O}_{H}\right)$, where $H$ is the Hilbert class field of the trace field of $\Gamma_{Q}([10$, Lemma 5.2.4]).

Recall that any parabolic in $\operatorname{PSL}(2, \mathbb{C})$ fixing $z=\beta / \gamma$ can be expressed as

$$
\left(\begin{array}{cc}
1+\beta \gamma & -\beta^{2} \\
\gamma^{2} & 1-\beta \gamma
\end{array}\right)
$$

Consequently, if such a parabolic in $\operatorname{PSL}(2, \mathbb{C})$ is also in $\operatorname{PSL}(2, \mathbb{A}),-\beta^{2}, \gamma^{2} \in \mathbb{A}$ and therefore $\beta, \gamma \in \mathbb{A}$.

Considering a Wirtinger presentation for $\Gamma_{K} \subset \Gamma_{Q} \subset \operatorname{PSL}(2, \mathbb{A})$, we may assume $\Gamma_{K}$ is generated by parabolics. Hence

$$
\Gamma_{K}=\left\langle\mu_{1}, \mu_{2}, \ldots, \mu_{n}\right\rangle, \quad \text { where } \mu_{i}=\left(\begin{array}{cc}
1+\beta_{i} \gamma_{i} & -\beta_{i}^{2} \\
\gamma_{i}^{2} & 1-\beta_{i} \gamma_{i}
\end{array}\right) \text { and } \beta_{i}, \gamma_{i} \in \mathbb{A} \text {. }
$$

Denote by $L$ the compositum of $H$ and $\mathbb{Q}\left(\beta_{i}, \gamma_{i} \mid 1 \leq i \leq n\right)$. We see that $H \subseteq L$ and $\mathcal{O}_{H} \subseteq \mathcal{O}_{L}$. Hence we have $\Gamma_{Q} \subset \operatorname{PSL}\left(2, \mathcal{O}_{H}\right) \subseteq \operatorname{PSL}\left(2, \mathcal{O}_{L}\right) \subset \operatorname{PSL}(2, \mathbb{A})$.

We claim that we can conjugate $\Gamma_{Q}$ such that $\Gamma_{Q}$ remains in $\operatorname{PSL}(2, \mathbb{A})$ with $\mu_{1}$ upper triangular. For ease of notation, we will suppress the subscript notation in the following argument. Note that here $\mu$ fixes $\beta / \gamma$. Therefore, it is sufficient to construct an element $h=\left(\begin{array}{ll}a & b \\ c & d\end{array}\right)$ with $a, b, c, d \in \mathbb{A}, a d-b c=1$ and $-d / c=\beta / \gamma$ (so $\left.h(\beta / \gamma)=\infty\right)$, since enforcing these properties ensures $h$ will be in $\operatorname{PSL}(2, \mathbb{A})$ (so $h \Gamma_{Q} h^{-1} \subset \operatorname{PSL}(2, \mathbb{A})$ ) and $h \mu h^{-1}$ will be upper triangular. The next paragraph establishes this claim by finding such an $h$.

If $\beta \mathcal{O}_{L}$ and $\gamma \mathcal{O}_{L}$ are comaximal, ie there exist $p, q \in \mathcal{O}_{L}$ such that $p \beta+q \gamma=1$, then $a=p, b=q, c=-\gamma$, and $d=\beta$ or $h=\left(\begin{array}{cc}p & q \\ -\gamma & \beta\end{array}\right)$ is a solution with the desired properties. If $\beta \mathcal{O}_{L}$ and $\gamma \mathcal{O}_{L}$ are not comaximal, then $\mathcal{I}=\langle\beta, \gamma\rangle$ is a proper ideal. In the case where $\mathcal{I}$ is principal, we may assume that $\beta=r \alpha, \gamma=s \alpha$ for some $\alpha \in \mathcal{O}_{L}$ 
or equivalently, $\mathcal{I}=\alpha \mathcal{O}_{L}$. Since $\mathcal{O}_{L}$ is a Dedekind domain, there exist $p, q \in \mathcal{O}_{L}$ such that $p \beta+q \gamma=\alpha$ and thus, $p r+q s=1$. Here, $a=p, b=q, c=-s$, and $d=r$ or $h=\left(\begin{array}{cc}p & q \\ -s & r\end{array}\right)$ yields a solution with the desired properties. In the case that $\mathcal{I}$ is not a principal ideal in $\mathcal{O}_{L}$, then we may pass to the Hilbert class field of $L$, say $E$, and apply the same argument since $\mathcal{I}$ is a principal ideal in $\mathcal{O}_{E}$.

The following lemma helps specify what values can go in the upper right entry of the meridian exhibited above.

Lemma 3.2 Let $\Gamma_{K}$ be a knot group in $\operatorname{PSL}(2, \mathbb{A})$ with an upper-triangular meridian $\mu=\left(\begin{array}{ll}1 & x \\ 0 & 1\end{array}\right)$. Then $x$ is a unit in $\mathbb{A}$. Furthermore, we may assume that $\mu=\left(\begin{array}{ll}1 & 1 \\ 0 & 1\end{array}\right)$.

Proof Since $\Gamma_{K}$ is finitely generated, we can attach all of the entries of the generators of $\Gamma_{K}$ to $\mathbb{Q}$ and get a finite extension of $\mathbb{Q}$. Denote this extension by $L$. Although this $L$ could be a proper subfield of that of the previous lemma, we still have the property that $\Gamma_{K} \subset \operatorname{PSL}\left(2, \mathcal{O}_{L}\right)$.

Assume $x$ is not a unit. Then $x \mathcal{O}_{L}$ is a proper ideal and we may say $x \mathcal{O}_{L}=\prod_{i=1}^{n} \mathcal{P}_{i}^{e_{i}}$, where each $\mathcal{P}_{i}$ is a maximal ideal in $\mathcal{O}_{L}$. Let $\mathcal{J}=\prod_{i=1}^{n} \mathcal{P}_{i}$ for the same $\mathcal{P}_{i}$.

Denote by $E$ the Hilbert class field of $L$. Then $\mathcal{J}=\alpha \mathcal{O}_{E}$ for some $\alpha \in \mathcal{O}_{E}$ and is principal. Since $E$ is an unramified extension of $\mathrm{L}$ and each of the $\mathcal{P}_{i}$ are maximal ideals in $\mathcal{O}_{L}, \mathcal{J}=\prod_{i=1}^{m} \mathcal{Q}_{i}$, where $\mathcal{Q}_{i} \neq \mathcal{Q}_{j}$ if $i \neq j$.

Let $g=\left(\begin{array}{ll}a & b \\ c & d\end{array}\right) \in \Gamma_{K}$ and denote by $h=\left(\begin{array}{cc}\sqrt{\alpha} & 0 \\ 0 & 1 / \sqrt{\alpha}\end{array}\right)$. Then

$$
h \cdot g \cdot h^{-1}=\left(\begin{array}{cc}
a & b \cdot \alpha \\
c / \alpha & d
\end{array}\right) .
$$

We claim $c / \alpha$ is in $\mathcal{O}_{E}$. To see this it suffices to show $c$ is in $\mathcal{J}$. There exists a homomorphism $f_{i}: \operatorname{PSL}\left(2, \mathcal{O}_{E}\right) \rightarrow \operatorname{PSL}\left(2, \mathcal{O}_{E} / \mathcal{Q}_{i}\right)$ for each prime ideal $\mathcal{Q}_{i}$. Under such a homomorphism $f_{i}\left(\Gamma_{K}\right)$ is trivial, since $f_{i}(\mu)$ is trivial and $\mu$ normally generates $\Gamma_{K}$. Therefore, $f_{i}(g)=\left(\begin{array}{ll}1 & 0 \\ 0 & 1\end{array}\right)$ and $c$ is in $\mathcal{Q}_{i}$ for each $\mathcal{Q}_{i}$. Hence $c$ is in $\mathcal{J}$.

Also, $h \cdot \mu \cdot h^{-1}=\left(\begin{array}{cc}1 & x \alpha \\ 0 & 1\end{array}\right)$. Note, that $x \alpha \mathcal{O}_{E}$ and $x \mathcal{O}_{E}$ factor into the same set of prime ideals, if we ignore the multiplicities of the factors. Hence the construction of $\mathcal{J}$ is independent of starting with $\Gamma_{K}$ or $h \Gamma_{K} h^{-1}$. In particular, $f_{i}\left(h \cdot \mu \cdot h^{-1}\right)$ is trivial. Therefore, by applying the above argument to $h \Gamma_{K} h^{-1}, c / \alpha \in \mathcal{J}$ as well.

Let

$$
V(g)=\min _{\left\{\mathcal{Q}_{i} \mid \mathcal{J} \subset \mathcal{Q}_{i}\right\}}\left\{v_{\mathcal{Q}_{i}}(c) \mid g=\left(\begin{array}{ll}
a & b \\
c & d
\end{array}\right)\right\} .
$$


Here $v_{\mathcal{Q}_{i}}(c)$ denotes the power of $\mathcal{Q}_{i}$ in the factorization of the ideal generated by $c$. By the above argument, we have established that $V(g)>0$ for all $g \in \Gamma_{K}$. We also note that $V\left(h \cdot g \cdot h^{-1}\right)=V(g)-1$.

There is an element $g^{\prime} \in \Gamma_{K}$ such that $V\left(g^{\prime}\right)=s$ is minimal. Thus $V\left(h^{s} \cdot g^{\prime} \cdot h^{-s}\right)=0$. Thus, for some $\mathcal{Q}_{i}$, the lower left entry of $h^{s} \cdot g^{\prime} \cdot h^{-s}$ is not in $\mathcal{Q}_{i}$ and so $f_{i}\left(h^{s} \cdot g^{\prime} \cdot h^{-s}\right)$ is nontrivial. However, $h^{s} \cdot \mu \cdot h^{-s}=\left(\begin{array}{cc}1 & x \alpha^{s} \\ 0 & 1\end{array}\right)$, implying $x \alpha^{s} \in \mathcal{Q}_{i}$ for all $i$. Furthermore, $h^{s} \cdot \mu \cdot h^{-s}$ normally generates $h^{s} \cdot \Gamma_{\boldsymbol{K}} \cdot h^{-s}$ and therefore $f_{i}\left(h^{s} \cdot \Gamma_{\boldsymbol{K}} \cdot h^{-s}\right)$ is trivial. This is a contradiction.

Finally, since $x$ is a unit, we may conjugate $\Gamma_{K}$ by $h^{\prime}=\left(\begin{array}{cc}1 / \sqrt{x} & 0 \\ 0 & \sqrt{x}\end{array}\right)$ so that there is a meridian of the form $\left(\begin{array}{ll}1 & 1 \\ 0 & 1\end{array}\right)$, while preserving the integrality of the representation.

Remark 3.3 As a consequence of the argument in Lemma 3.2 with $\Gamma_{K}$ in PSL(2, $\left.\mathcal{O}_{L}\right)$ (with $L$ defined as in the proof of Lemma 3.1), the meridian

$$
\mu=\left(\begin{array}{cc}
1+\beta \gamma & -\beta^{2} \\
\gamma^{2} & 1-\beta \gamma
\end{array}\right)
$$

must have the property that $\beta \mathcal{O}_{L}$ and $\gamma \mathcal{O}_{L}$ are comaximal. Otherwise, after conjugation the upper-triangular meridian will not have a unit in the upper right entry.

\section{Proof of Theorem 1.1}

In this section, we prove Theorem 1.1. In the proof, we assume that $S^{3}-K$ cyclically covers an orbifold with a torus cusp, which we denote by $Q_{T}$ throughout the section. Also, we will use $\Gamma_{T}=\pi_{1}^{\text {orb }}\left(Q_{T}\right)$ and denote by $P_{T}$ the peripheral subgroup of $\Gamma_{T}$. The main argument is that $Q_{T}$ cannot admit multiple cyclic fillings because $Q_{T}$ must be a relatively high-degree cover of any rigid cusped orbifold.

We say the isotropy graph $\Omega$ of an orbifold $Q$ has a loop if there is an edge in $\Omega$ that begins and ends at the same vertex. If $Q$ does not have a torus cusp and is covered by a knot complement, it is a consequence of the cusp-killing homomorphism that loops in $\Omega$ must begin and end at the cusp.

We note that Propositions 4.1 and 4.2 are used in the proof of Theorem 1.2. Also, the figures in the remainder of the paper involving $Q$ and its isotropy graph will represent the cusp as a bolded point and the base space of $Q$ should be thought of as $D^{3} \cong S^{3}-\{\mathrm{pt}\}$. Thus the degree of cusp will be 3 if the cusp is rigid and 4 if the cusp is $S^{2}(2,2,2,2) \times[0, \infty)$. All other vertices will be degree 3 and we refer to them as internal vertices for convenience.

We begin by classifying the possible abelian quotients of rigid cusped orbifolds covered by hyperbolic knot complements. 
Proposition 4.1 Let $S^{3}-K$ be a hyperbolic knot complement that covers an orbifold $Q$.

(1) If $Q$ has a $S^{2}(2,3,6)$ cusp, then $\mathbb{Z} / 2 \mathbb{Z}$ surjects onto $\Gamma_{Q}^{a b}$. Furthermore, $\Gamma_{Q}^{a b} \cong$ $\mathbb{Z} / 2 \mathbb{Z}$ if and only if there is a 2 -fold cover $Q^{\prime}$ of $Q$ with a $S^{2}(3,3,3)$ cusp.

(2) If $Q$ has a $S^{2}(3,3,3)$ cusp, then $\mathbb{Z} / 3 \mathbb{Z} \times \mathbb{Z} / 3 \mathbb{Z}$ surjects onto $\Gamma_{Q}^{a b}$.

(3) If $Q$ has a $S^{2}(2,4,4)$ cusp, $\Gamma_{Q}^{a b}$ is trivial, $\mathbb{Z} / 2 \mathbb{Z}, \mathbb{Z} / 2 \mathbb{Z} \times \mathbb{Z} / 2 \mathbb{Z}$ or $\mathbb{Z} / 4 \mathbb{Z}$. Furthermore, $\Gamma_{Q}^{a b} \cong \mathbb{Z} / 4 \mathbb{Z}$ if and only if the isotropy graph of $Q$ has a loop.

Proof We note that $\Gamma_{Q}=P_{Q} \cdot \Gamma_{K}$. Therefore, we claim that

$$
\Gamma_{Q}=\left\langle\left\langle t=\left(\begin{array}{ll}
1 & 1 \\
0 & 1
\end{array}\right), r=\left(\begin{array}{cc}
\ell & 0 \\
0 & \ell^{-1}
\end{array}\right)\right\rangle\right\rangle_{P_{Q} \cdot \Gamma_{K}},
$$

where $r$ is an elliptic element of order 3, 4 or 6 depending on the cusp type. The claim follows from the fact that $r$ and $t$ are generators for $P_{Q}$ and $P_{Q}$ contains a meridian of $\Gamma_{K}$.

First assume $Q$ has a $S^{2}(2,3,6)$ cusp. Then $\ell=e^{i \pi / 6}$. Since $P_{Q}$ abelianizes to $\mathbb{Z} / 6 \mathbb{Z}$, $\Gamma_{Q}^{a b}$ is a quotient of $\mathbb{Z} / 6 \mathbb{Z}$. Also, the torsion element of order 6 is connected to an interior vertex with the isotropy group a $D_{6}$ (dihedral group of order 12). Under the abelianization of this isotropy group, the element of order 6 maps to an element of order 2. Thus $\Gamma_{Q}^{a b}$ is a quotient of $\mathbb{Z} / 2 \mathbb{Z}$. If $\Gamma_{Q}^{a b} \cong \mathbb{Z} / 2 \mathbb{Z}$, then $Q$ has a 2 -fold cover $Q^{\prime}$ such that $\Gamma_{Q^{\prime}}$ is the kernel of the abelianization. Thus $Q^{\prime}$ has only 3-torsion on the cusp.

Next, assume $Q$ has a $S^{2}(3,3,3)$ cusp. In this case, $\ell=e^{2 i \pi / 3}$. Then $\Gamma_{Q}^{a b}$ is a quotient of $\mathbb{Z} / 3 \mathbb{Z} \times \mathbb{Z} / 3 \mathbb{Z}$, the abelianization of the peripheral subgroup (see Section 2.1).

Finally, assume $\mathrm{Q}$ has a $S^{2}(2,4,4)$ cusp. Then $\ell=e^{i \pi / 4}$. In this case, $\Gamma_{Q}^{a b}$ is a quotient of the abelianization of $P_{Q}$ that is $\mathbb{Z} / 2 \mathbb{Z} \times \mathbb{Z} / 4 \mathbb{Z}$.

Consider an edge $e$ labeled by 4-torsion that connects the cusp $c$ to another vertex $x$. Then $x$ is either the cusp itself, or it corresponds to a point fixed by an isotropy group isomorphic to $D_{4}$ or $S_{4}$ (see Section 2.2).

Case 1: $e$ is a loop In this case, $e$ connects the cusp back to itself. Then we see from a Wirtinger presentation for $\Gamma_{Q}$ coming from the isotropy graph that $\Gamma_{Q}^{a b}$ is either $\mathbb{Z} / 4 \mathbb{Z}$ or $\mathbb{Z} / 2 \mathbb{Z} \times \mathbb{Z} / 4 \mathbb{Z}$. In the first case, the image of a peripheral element of order 4 generates $\Gamma_{Q}^{a b}$. In the second case, both a peripheral element of order 4 and a peripheral element of order 2 generate $\Gamma_{Q}^{a b}$. However, in order for a peripheral element of order 2 to map nontrivially under $\pi: \Gamma_{Q} \rightarrow \Gamma_{Q}^{a b}$, we would need a path labeled by 
only even numbers that starts and ends at the cusp (by the cusp-killing homomorphism) and includes the 2-torsion on the cusp. Such a cycle would also include the 4-torsion on the cusp contradicting our assumption that $e$ is a loop.

Case 2: $\boldsymbol{x}$ corresponds to $\boldsymbol{D}_{\mathbf{4}}$ or $\boldsymbol{S}_{\mathbf{4}}$ In this case, the isotropy graph of $Q$ does not contain a loop. Under the abelianizations of these groups, elements of order 4 are mapped to elements of order 2 . Under the abelianization of the cusp, the peripheral elements of order 4 all have the same order in $\Gamma_{Q}^{a b}$. Therefore, $\mathbb{Z} / 2 \mathbb{Z} \times \mathbb{Z} / 2 \mathbb{Z}$ surjects onto $\Gamma_{Q}^{a b}$.

In the following propositions and lemmas, we appeal to notation and definitions from [5]. In that paper, the authors define an orbilens space to be the quotient of $S^{3} / \Delta$, where $\Delta$ is a finite cyclic subgroup of $\mathrm{SO}(4, \mathbb{R})$. As noted in that paper, for any orbilens space $L,|L|$ is a lens space $L(p, q)$. Furthermore, there exists a Heegaard splitting of $|L|$ such that $L$ decomposes into two pieces each of which is the quotient of solid torus under rotation about its core. Using $p, q$ to denote the underlying lens space and $m, n$ to denote the orders of these rotations, we use the notation $L(p, q ; m, n)$ to denote an orbilens space. Finally, by [5, Proposition 4.13] if two knot complements are cyclically commensurable, they both cover the complement knot in an orbilens space.

The lower bound on the degree of a manifold cover exhibited by this proposition will be used in the next section. However, the classification of knot complements in orbilens spaces that 4-fold cover rigid cusped orbifolds with loops will be useful in this section.

Proposition 4.2 Let $M$ be a hyperbolic manifold. If $p: M \rightarrow Q$ with a $S^{2}(2,4,4)$ cusp, the isotropy graph for $Q$ has a loop labeled 4 , and $M$ is covered by a knot complement, then $\operatorname{deg}(p) \geq 24$. Furthermore, if there is a loop labeled 4 , then there exists $f: Q_{T} \rightarrow Q$, where $Q_{T}$ is a knot complement in an orbilens space $O$ and $\operatorname{deg}(f)=4$, where the singular locus of $O$ is two unknotted circles.

Proof Assume that $Q$ is covered by a knot complement and has a loop labeled 4 in its isotropy graph (see Figure 4 for a possible example). Then by Proposition 4.1, $\Gamma_{Q}^{a b}$ is $\mathbb{Z} / 4 \mathbb{Z}$. Hence there is a unique orbifold that is a 2 -fold cover of $Q$. We call this orbifold $Q^{\prime}$. Since $\Gamma_{Q^{\prime}}$ is characteristic in $\Gamma_{Q}$ and the abelian quotient of $\Gamma_{Q}$ is generated by $P_{Q}, P_{Q^{\prime}}$ is characteristic in $P_{Q}$. Thus $P_{Q^{\prime}}$ and $P_{Q}$ have the same maximal parabolic subgroup and $P_{Q^{\prime}}$ contains a meridian $\mu$ of $\Gamma_{K}$. In particular, $\langle\langle\mu\rangle\rangle_{\Gamma_{K}} \subset \Gamma_{Q^{\prime}}$. Therefore, $S^{3}-K$ covers $Q^{\prime}$. Again using the fact the $\Gamma_{Q}^{a b}$ is $\mathbb{Z} / 4 \mathbb{Z}$ and that the isotropy graph of $Q$ has a loop labeled $4, \Gamma_{Q^{\prime}}$ is the kernel of the map from $\Gamma_{Q}$ to $\mathbb{Z} / 2 \mathbb{Z}$, all elements of order 4 in $P_{Q}$ are nontrivial in the image of such a map, and all elements of order 2 in $P_{Q}$ map trivially. Thus $Q^{\prime}$ has a $S^{2}(2,2,2,2)$ 


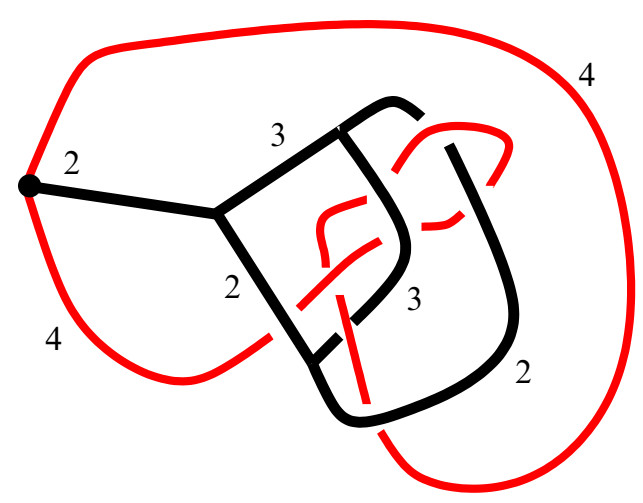

Figure 4: A possible isotropy graph of an orbifold with a $S^{2}(2,4,4)$ cusp

cusp. By [17, Proposition 9.2], this cover $p^{\prime}: S^{3}-K \rightarrow Q^{\prime}$ is regular. Furthermore, by the proof of that argument $\Gamma_{Q^{\prime}} / \Gamma_{K}$ is dihedral and there is an index-2 subgroup of $\Gamma_{Q^{\prime}}, \Gamma_{T}$, that preserves the orientation of the meridians of $\Gamma_{K}$. Thus there is a unique 2-fold cover of $Q^{\prime}$ that has a torus cusp. While keeping consistent with our previous conventions, we denote this orbifold by $Q_{T}$. We know that $S^{3}-K$ covers $Q_{T}$ since $\Gamma_{K}$ is generated by parabolics and $\left[P_{Q}: P_{T}\right]=4$, which implies that all parabolic elements of $\Gamma_{Q}$ are contained in $\Gamma_{T}$.

Since $Q_{T} \cong\left(S^{3}-K\right) / Z$ for some cyclic group $Z$ of isometries of $S^{3}$, we see that $Q_{T}$ is the complement of a knot in an orbilens space. By [5, Lemma 3.1], the base space of $Q_{T}$ is a lens space, $Z$ preserves a Hopf fibration of $S^{3}$ and isotropy graph of $Q_{T}$ is a set of circles $C$ such that $C$ lifts to 0,1 or 2 great circles in $S^{3}$. We can obtain $Q^{\prime}$ as the quotient of $Q_{T}$ by a symmetry $\tau$ that fixes points on the cusp of $Q_{T}$. With a slight abuse of notation, we may also view $\tau$ as an isometry of $S^{3}$ that fixes points in $S^{3}$ (or $Q^{\prime} \cong\left(S^{3}-K\right) /\langle Z, \tau\rangle$ ). The fixed-point set of $\tau$ is also a great circle by the positive resolution of the Smith conjecture (see Morgan [14]). Hence the fixed-point set of $\tau$ must intersect each circle of $C$ in 0 or 2 points. Therefore, the isotropy graph for $Q^{\prime}$ contains 0,2 or 4 internal vertices. The next three paragraphs deal with each case respectively.

The isotropy graph for $Q^{\prime}$ cannot contain 0 vertices because that would imply that the isotropy graph for $Q$ only had vertices labeled by Klein 4 groups. Such a graph would be nontrivial under the cusp-killing homomorphism. Since the 2 -fold cover of the loop labeled by 4 is a loop labeled by 2 , the possible graphs as defined up to graph isomorphism type can be seen in Figure 5.

We claim that the isotropy graph for $Q^{\prime}$ cannot contain 2 internal vertices as well. First, notice that the isotropy graph for $Q^{\prime}$ has an edge $e$ labeled by 2 -torsion with both 


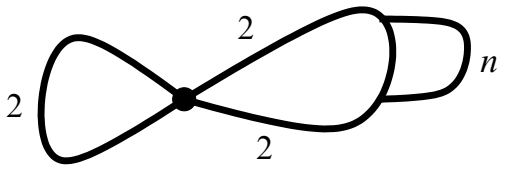

(a) A graph with 2 internal vertices

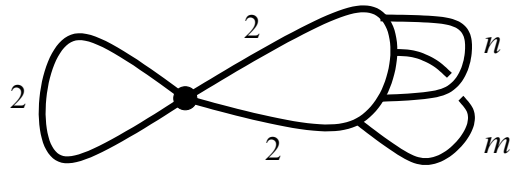

(b) A graph with 4 internal vertices

Figure 5: The possible isotropy graphs $Q^{\prime}$

endpoints on the cusp. Thus the isotropy graph of $Q$ takes the form of Figures 6(a), 6(b) and 6(c). In the latter two cases, $\pi_{1}^{\text {orb }}(Q)$ is nontrivial under the cusp-killing homomorphism and therefore $Q$ cannot be covered by a knot complement. In the first case, we cannot close up the isotropy graph. Therefore, no such orbifold $Q$ can be covered by a knot complement.

Finally, if $Q^{\prime}$ contains 4 internal vertices, then $Q_{T}$ is the complement of a knot in an orbilens space with an isotropy graph consisting of two unknotted circles. Since the circles are labeled by $m$-torsion and $n$-torsion with $m, n \geq 2$ and $(m, n)=1, S^{3}-K$ is at least a 6 -fold cover of $Q_{T}$ and therefore at least a 24 -fold cover of $Q$. This completes the proof.

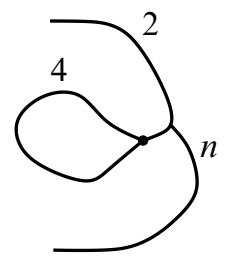

(a) The first case

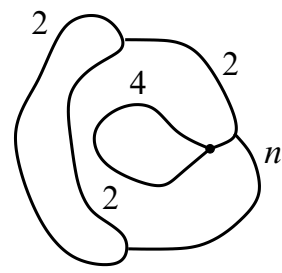

(b) The second case

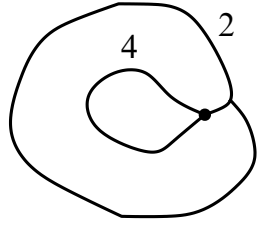

(c) The third case

Figure 6: The three cases for the isotropy graph in Proposition 4.2

Lemma 4.3 Let $S^{3}-K$ is small hyperbolic knot complement that covers an orbifold $Q_{T}$ with a torus cusp. Furthermore assume $Q_{T}$ covers $Q$ with a rigid cusp with covering degree $d$.

(1) If $Q$ has a $S^{2}(3,3,3)$ cusp, then $d>3$.

(2) If $Q$ has a $S^{2}(2,3,6)$ cusp, then $d>6$.

(3) If $Q$ has a $S^{2}(2,4,4)$ cusp, then $d>4$ or $Q_{T} \cong L(p, q: n, m)-K^{\prime}$ for some embedded knot $K^{\prime}$ and some $n \neq 1$ and $m \neq 1$. 
Proof Case 1: $Q$ has a $\boldsymbol{S}^{\mathbf{2}}(\mathbf{3}, \mathbf{3}, \mathbf{3})$ cusp First note that $d \geq 3$ since $Q$ has 3torsion on the cusp and $Q_{T}$ has a torus cusp. If $d=3$, then all isotropy groups must lift to cyclic groups. From inspection of the possible isotropy groups, all groups must be $D_{3}$ since no other candidates have index 3 cyclic groups. In this case, $\Gamma_{Q}$ would be nontrivial under the cusp-killing map, so it cannot be covered by a knot complement. Thus $d>3$.

Case 2: $\boldsymbol{Q}$ has a $S^{\mathbf{2}}(\mathbf{2}, 3,6)$ cusp By a similar argument to that above $d \geq 6$. If $d=6$, then $\left[\Gamma_{Q}: \Gamma_{T}\right]=6$ and there is a homomorphism from $\Gamma_{Q}$ to $S_{6}$ via left multiplication on the cosets of $\Gamma_{T}$. Denote by $r$ an element of 6-torsion that fixes a point on the cusp. Then $\Gamma_{Q}=\left\{\Gamma_{T}, r \cdot \Gamma_{T}, r^{2} \cdot \Gamma_{T}, r^{3} \cdot \Gamma_{T}, r^{4} \cdot \Gamma_{T}, r^{5} \cdot \Gamma_{T}\right\}$. Hence $r$ maps to an element of order 6 and the image of $\Gamma_{Q}$ is not a subgroup of $A_{6}$. Therefore, $\Gamma_{Q}$ admits a $\mathbb{Z} / 2 \mathbb{Z}$ quotient and a unique subgroup $\Gamma_{Q^{\prime}}$ of index 2 which contains $\Gamma_{T}$. As seen in Proposition 4.1, such a group corresponds to an orbifold $Q^{\prime}$ with a $S^{2}(3,3,3)$ cusp and so we have reduced the problem to the previous case.

Case 3: $Q$ has a $S^{\mathbf{2}}(\mathbf{2}, 4,4)$ cusp If $d=4$, then the 4-torsion either forms a loop, is connected to a $D_{4}$ or $S_{4}$ isotropy group. The first case is covered by Proposition 4.2. In the second case, some 2-torsion must survive the lift. However, the 2-torsion on the cusp must be a part of a $D_{2 n+1}$ in order to lift to a cyclic group and allow for $\Gamma_{Q}$ to be nontrivial under the cusp-killing map. In the final case, $S_{4}$ has no cyclic subgroups of index 4.

Before providing the proof Theorem 1.1, we establish one more technical lemma that exploits the properties of integral representations and presents the maximal abelian subgroup of $P_{Q}$ in an especially useful manner.

Lemma 4.4 If $Q$ is a rigid cusped orbifold such that $Q$ is covered by a knot complement $S^{3}-K$ and $\Gamma_{Q} \subset \operatorname{PSL}(2, \mathbb{A})$, then, up to conjugation in $\operatorname{PSL}(2, \mathbb{A}), P_{Q}$ is of the form

$$
\left\langle\left(\begin{array}{cc}
\zeta_{m} & x \\
0 & \zeta_{m}^{-1}
\end{array}\right),\left(\begin{array}{ll}
1 & 1 \\
0 & 1
\end{array}\right),\left(\begin{array}{cc}
1 & \zeta_{m}^{2} \\
0 & 1
\end{array}\right)\right\rangle
$$

where $x \in \mathbb{A}$ and $\zeta_{m}$ is a primitive $m^{\text {th }}$ root of unity. Furthermore, $m=6$ if $Q$ has a $S^{2}(3,3,3)$ cusp, $m=8$ if $Q$ has a $S^{2}(2,4,4)$ cusp, and $m=12$ if $Q$ has a $S^{2}(2,3,6)$ cusp.

Proof First, the case where $Q$ has a $S^{2}(3,3,3)$ cusp. By assumption, $Q$ is covered by a knot complement $S^{3}-K$ and $\Gamma_{K} \subset \Gamma_{Q} \subset \operatorname{PSL}(2, \mathbb{A})$. Applying Lemma 3.2 to these assumptions, we may also assume that there is a meridian $\mu$ of $\Gamma_{K}$ with 
$\mu=\left(\begin{array}{ll}1 & 1 \\ 0 & 1\end{array}\right)$. In addition, $P_{Q}$ contains a rotation of order 3 of the form $\left(\begin{array}{cc}\zeta_{6} & x \\ 0 & \zeta_{6}^{-1}\end{array}\right)$, where $x \in \mathbb{A}$. The rotation and $\mu$ generate a subgroup in $P_{Q}$ of the form

$$
P^{\prime}=\left\langle\left(\begin{array}{cc}
\zeta_{6} & x \\
0 & \zeta_{6}^{-1}
\end{array}\right),\left(\begin{array}{ll}
1 & 1 \\
0 & 1
\end{array}\right),\left(\begin{array}{cc}
1 & \zeta_{6}^{2} \\
0 & 1
\end{array}\right)\right\rangle .
$$

We claim that $P^{\prime}=P_{Q}$. First,

$$
P_{Q}=\left\langle\left(\begin{array}{cc}
\zeta_{6} & x \\
0 & \zeta_{6}^{-1}
\end{array}\right),\left(\begin{array}{ll}
1 & y \\
0 & 1
\end{array}\right),\left(\begin{array}{cc}
1 & y \zeta_{6}^{2} \\
0 & 1
\end{array}\right)\right\rangle
$$

for some $y \in \mathbb{A}$. Since $\mu \in P_{Q}$, we have that $1=y\left(r+s \cdot \zeta_{6}^{2}\right)$, where $r, s \in \mathbb{Z}$, and so $y,\left(r+s \cdot \zeta_{6}^{2}\right)$ are units in $\mathbb{A}$. It also follows directly from this computation that $y \in \mathbb{Q}(\sqrt{-3})$, and so $y=\zeta_{6}^{j}$ for some $j$. This proves the claim, completing the proof of the lemma in this case.

The cases where $Q$ has a $S^{2}(2,4,4)$ cusp or a $S^{2}(2,3,6)$ cusp each follow by nearly identical arguments where $\zeta_{6}$ is replaced by $\zeta_{8}$ and $\zeta_{12}$ respectively.

We are now ready to prove Theorem 1.1.

Proof of Theorem 1.1 Let $S^{3}-K$ be a small knot complement that covers an orbifold $Q$ with a rigid cusp. Since $S^{3}-K$ is small, $\Gamma_{K}$ admits integral traces (see [4]). Also, all groups commensurable with $\Gamma_{K}$ admit integral traces. In particular, $\Gamma_{Q}$ admits integral traces. Hence, by [10, Lemma 5.2.4], there is a representation of $\Gamma_{Q}$ into $\operatorname{PSL}(2, \mathbb{A})$.

Assume $S^{3}-K^{\prime}$ is a knot complement that is cyclically commensurable with $S^{3}-K$. By [5, Proposition 4.7], both knot complements cover an orbifold $Q_{T}$, which is a knot complement in an orbilens space covered by both $S^{3}-K^{\prime}$ and $S^{3}-K$. The remainder of the proof will show that such an orbifold $Q_{T}$ cannot exist.

Since the commensurability class of $S^{3}-K$ contains at least two knot complements, $S^{3}-K$ is nonarithmetic (see [21]), and so we may assume that $Q$ is the orientable commensurator quotient. Therefore, $S^{3}-K, S^{3}-K^{\prime}$ and $Q_{T}$ cover $Q$. By consideration of the representation for $\Gamma_{Q}$ constructed in Lemma 3.1, $\Gamma_{T}$ admits an integral representation such that $P_{T}$ is upper triangular and contains two distinct knot meridians $\mu, \mu^{\prime}$ corresponding to $S^{3}-K$ and $S^{3}-K^{\prime}$ respectively. Moreover, by Lemma 3.2, $\mu$ and $\mu^{\prime}$ have units in their upper right entries. By Lemma 4.4, if $Q$ has a $S^{2}(3,3,3)$ or $S^{2}(2,3,6)$ cusp, the maximal abelian subgroup of $P_{Q}$ is of the form $\left\langle\left(\begin{array}{ll}1 & 1 \\ 0 & 1\end{array}\right),\left(\begin{array}{ll}1 & \omega \\ 0 & 1\end{array}\right)\right\rangle$ (where $\left.\omega^{2}+\omega+1=0\right)$, and if $Q$ has a $S^{2}(2,4,4)$ cusp, the maximal abelian subgroup of $P_{Q}$ is of the form $\left\langle\left(\begin{array}{ll}1 & 1 \\ 0 & 1\end{array}\right),\left(\begin{array}{ll}1 & i \\ 0 & 1\end{array}\right)\right\rangle$. Regardless of the cusp 
type, the property that $P_{T}$ contains two elements with units in the off diagonal entries implies $P_{T}$ is the minimal index abelian subgroup of $P_{Q}$. Since the index of $P_{T}$ in $P_{Q}$ is the degree of the cover $p: Q_{T} \rightarrow Q$, this contradicts Lemma 4.3 unless $Q$ has a $S^{2}(2,4,4)$ cusp and $Q_{T} \cong L(p, q ; m, n)-K^{\prime}$. In this case, $m \neq 1$ and $n \neq 1$. However, by [5, Theorem 1.8], such a $Q_{T}$ cannot be covered by two knot complements. This completes the proof.

\section{Proof of Theorem 1.2}

In this section, we prove Theorem 1.2. The first step of the proof involves two arguments that orbifolds with certain prescribed cusp volumes cannot be covered by knot complements. The second ingredient is a lower bound on the degree of the covering $p: M \rightarrow Q$, where $M$ is a manifold covered by a small knot complement and $Q$ is a rigid cusped orbifold. The third part of the proof establishes an upper bound on $\operatorname{deg}(p)$ by combining the six theorem of Agol and Lackenby (see [2;9]), the lemmas of Section 3, and Adams' classification of small cusp volume orbifolds (see Theorem 2.1).

In the following proofs, we will identify $\mathbb{H}^{3}$ with $\left\{z+c j \in \mathbb{H} \mid z \in \mathbb{C}, c>0, j^{2}=-1\right\}$ (upper-half space) and $\partial \mathbb{H}^{3}$ with $\mathbb{C} \cup\{\infty\}$. Here we denote by $B_{x}$ the horoball that is tangent to $\partial \mathbb{H}^{3}$ at $x$. Lemma 5.1 follows directly from Adams' paper on small volume orbifolds (see [1]). However, the explicit representation of $\Gamma_{Q}$ is unnecessary for the results of that paper. Below, we give a relevant summary of Adams' work in order to provide this representation, which is the key component of the proof of Lemma 5.2.

Lemma 5.1 (Adams 1991) The orbifold fundamental group of the orbifold $Q$ with cusp volume $\sqrt{21} / 24$ admits a representation into $\operatorname{PSL}(2, \mathbb{C})$ given by

$$
\Gamma_{Q}=\left\langle t=\left(\begin{array}{cc}
1 & \sqrt[4]{7} \\
0 & 1
\end{array}\right), r=\left(\begin{array}{cc}
\ell & 0 \\
0 & \ell^{-1}
\end{array}\right), \gamma=\left(\begin{array}{cc}
0 & i \cdot b \\
\frac{i}{b} & 0
\end{array}\right)\right\rangle,
$$

where $\ell=(\sqrt{3}+i) / 2$ and $b=\sqrt{5+i \sqrt{3}} / \sqrt{2 \sqrt{7}}$.

Proof Let $Q$ be a 3 -orbifold with a $S^{2}(2,3,6)$ cusp and cusp volume $\sqrt{21} / 24$. A diagram of the horoballs associated to $Q$ first appeared in Adams' paper (see [1, Figure 5]). It is included here as Figure 7 for the sake of completeness. Furthermore, following the discussion of this horoball diagram in [1], we use the following notation: $O=0, D=\sqrt[4]{7}, X=(5+i \sqrt{3}) / 2 \sqrt{7}$ and $Y=\sqrt[4]{7} / 2+i(\sqrt[4]{7} / 2 \sqrt{3})$.

In this figure, there are four horoballs pictured. Following the description of this diagram from Adams' work, the horoballs $B_{O}$ and $B_{D}$ are of Euclidean diameter 1 and maximal 
in the sense that they are tangent to the horoball based at $\infty$. The horoball $B_{X}$ has Euclidean diameter $1 / \sqrt{7}$ and the horoball $B_{Y}$ has Euclidean diameter $\frac{3}{7}$. The line segment $\overline{O Y}$ has length $w=\sqrt[4]{7} / \sqrt{3}$ while the line segment $\overline{O X}$ has length $1 / \sqrt[4]{7}$. Note that $\sqrt[4]{7} / \sqrt{3} \approx 0.939104416<1$.

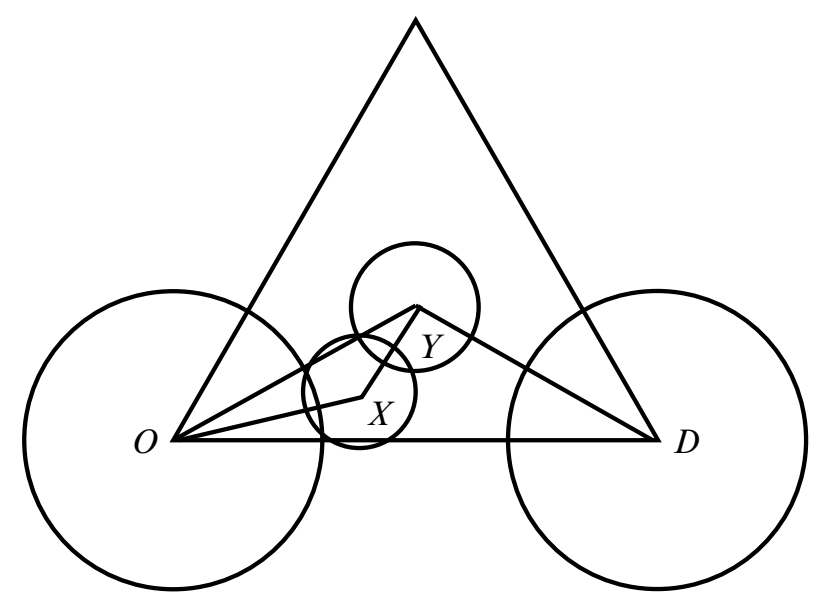

Figure 7: A horoball diagram for $Q$

Under Adams' description of $Q$, we see that $\Gamma_{Q}$ contains a parabolic element $t$ such that $t(\infty)=\infty$ and $t(0)=\sqrt[4]{7}$. In addition, $\Gamma_{Q}$ contains an order 6 rotation $r$ that fixes 0 and $\infty$. Finally, as Adams notes, all horoballs of Euclidean diameter 1 are equivalent under the action of $P_{Q}$. By [1, Lemma 1.2], there is an element $\gamma$ that exchanges 0 and $\infty$ while sending $\sqrt[4]{7}$ to $(5+i \sqrt{3}) /\left(2 \cdot \sqrt[4]{7^{3}}\right)$. Therefore,

$$
t=\left(\begin{array}{cc}
1 & \sqrt[4]{7} \\
0 & 1
\end{array}\right), \quad r=\left(\begin{array}{cc}
\ell & 0 \\
0 & \ell^{-1}
\end{array}\right) \quad \text { and } \quad \gamma=\left(\begin{array}{cc}
0 & i \cdot b \\
\frac{i}{b} & 0
\end{array}\right),
$$

where $\ell=(\sqrt{3}+i) / 2$ and $b=\sqrt{5+i \sqrt{3}} / \sqrt{2 \sqrt{7}}$.

The isometric sphere of $\gamma$ is of radius 1 and centered at 0 (see Figure 8). Hence the isometric sphere for $t \cdot \gamma \cdot t^{-1}$ is of radius 1 and centered at $\sqrt[4]{7}$. Let $\Gamma=\langle t, r, \gamma\rangle$. Since these two isometric spheres bound a fundamental domain for $\Gamma$ away from $\mathbb{C}, \Gamma$ has finite covolume. Also, since the cusp covolume of $\Gamma$ is $\sqrt{21} / 24,\left[\Gamma_{Q}: \Gamma\right]=1$.

The following lemma exploits the arithmetic properties of representation of $\Gamma_{Q}$ from the previous lemma in order to show that an orbifold with cusp volume $\sqrt{21} / 24$ cannot be covered by a knot complement. 
Lemma 5.2 (1) Any orbifold with a $S^{2}(3,3,3)$ cusp and cusp volume $\frac{\sqrt{21}}{12}$ cannot be covered by a knot complement.

(2) Any orbifold with a $S^{2}(2,3,6)$ cusp and cusp volume $\frac{\sqrt{21}}{24}$ cannot be covered by a knot complement.

Proof First, we appeal to Adams' characterization of orbifolds of small cusp volume (see Section 2.1) to reduce to case (2) as any orbifold with a $S^{2}(3,3,3)$ cusp and cusp volume $\sqrt{21} / 12$ covers an orbifold with $S^{2}(2,3,6)$ cusp and cusp volume $\sqrt{21} / 24$.

Let $Q$ be an orbifold with cusp volume $\frac{\sqrt{21}}{24}$, then as seen in Lemma 5.1,

$$
\Gamma_{Q}=\left\langle t=\left(\begin{array}{cc}
1 & \sqrt[4]{7} \\
0 & 1
\end{array}\right), r=\left(\begin{array}{cc}
\ell & 0 \\
0 & \ell^{-1}
\end{array}\right), \gamma=\left(\begin{array}{cc}
0 & i \cdot b \\
\frac{i}{b} & 0
\end{array}\right)\right\rangle,
$$

where $\ell=(\sqrt{3}+i) / 2$ and $b=\sqrt{5+i \sqrt{3}} / \sqrt{2 \sqrt{7}}$.

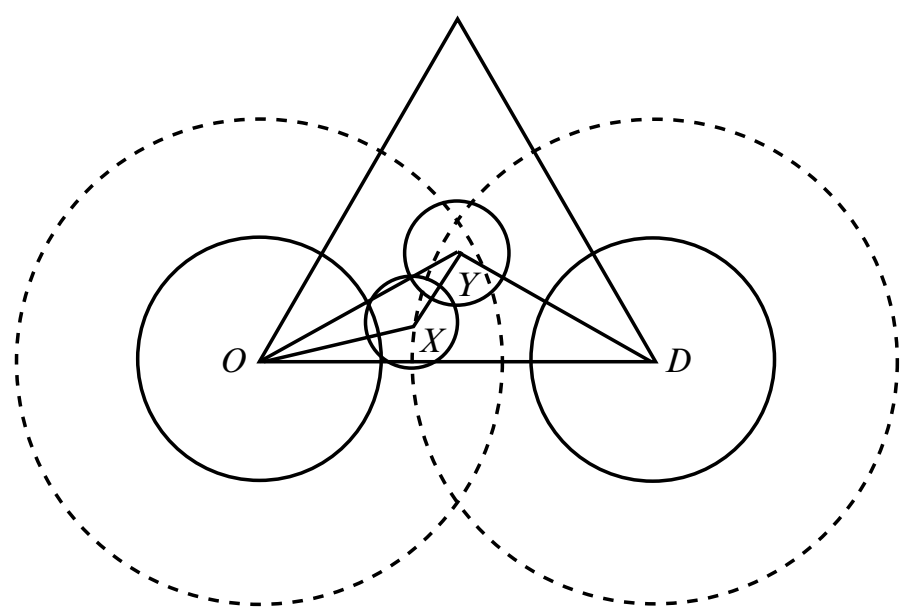

Figure 8: A horoball diagram for $Q$ with the isometric spheres represented by dotted curves around 0 and $D$

Let $\lambda=\frac{1}{\sqrt{b}}$ and $g=\left(\begin{array}{cc}\lambda & 0 \\ 0 & \lambda^{-1}\end{array}\right)$. Then

$$
g \cdot \Gamma_{Q} \cdot g^{-1}=\left\langle t^{\prime}=\left(\begin{array}{ll}
1 & \alpha \\
0 & 1
\end{array}\right),\left(\begin{array}{cc}
\ell & 0 \\
0 & \ell^{-1}
\end{array}\right), \gamma^{\prime}=\left(\begin{array}{ll}
0 & i \\
i & 0
\end{array}\right)\right\rangle,
$$

where $\alpha=\sqrt{14 /(5+i \sqrt{3})}$. Note the minimal polynomial for $\alpha$ is $q^{4}-5 q^{2}+7=$ 0 . Thus $\alpha$ is an algebraic integer, but not a unit since the constant term of this polynomial is not 1 . Under this integral representation of $\Gamma_{Q}$, there are upper-triangular 
parabolic elements all of which have nonunits in their off diagonal entries. Hence a knot complement covering $Q$ would contradict Lemma 3.2.

The following proposition specifies which finite groups can act on a point of tangency between two horoballs.

Proposition 5.3 Consider a maximal horoball packing corresponding to an orbifold with a rigid cusp. Denote by $B_{x}$ the horoball centered $x$ and denote by $B_{\infty}$ the horoball at $\infty$. If $y$ is the point of tangency of $B_{x}$ and $B_{\infty}$ and $y$ is fixed by an element $\gamma \in \mathrm{Stab}_{\infty}$, then the isotropy group of $\mathrm{y}$ is $C_{n}$ or $D_{n}$, where $n=2,3,4,6$.

Proof First $\gamma$ is order 2, 3, 4 or 6 because it fixes $\infty$. If Stab $y$ is cyclic, we are done. Thus we can assume the isotropy group of $y$ has elements fixing two distinct axes through $y$.

Denote by $\gamma^{\prime}$ be an element of the isotropy group of $y$ such that the axis fixed by $\gamma^{\prime}$ intersects the axis fixed by $\gamma$ at the smallest (nonzero) angle possible. Denote this angle by $\alpha$. If $\alpha=\pi / 2$, then $\left\langle\gamma, \gamma^{\prime}\right\rangle$ is dihedral (see Section 2.2). Hence we may assume that $\alpha<\pi / 2$. Therefore, $\gamma^{\prime}$ fixes points inside of $B_{\infty}$. However $\gamma^{\prime}\left(B_{\infty}\right) \cap B_{\infty}=\varnothing$ and $\gamma^{\prime}$ does not fix $\infty$, which is a contradiction.

We are now ready to prove the following lemma.

Lemma 5.4 (1) Any orbifold $Q$ with a $S^{2}(3,3,3)$ cusp and cusp volume $\sqrt{3} / 4$ such that $\Gamma_{Q}$ admits integral traces cannot be covered by a knot complement.

(2) Any orbifold $Q$ with a $S^{2}(2,3,6)$ cusp and cusp volume $\sqrt{3} / 8$ such that $\Gamma_{Q}$ admits integral traces cannot be covered by a knot complement.

Proof We begin by assuming that $Q$ has a $S^{2}(3,3,3)$ cusp and has cusp volume $\sqrt{3} / 4$, and $\Gamma_{Q}$ admits integral traces. Consider a horoball diagram for the fundamental domain of $Q$ viewed from the point at $\infty$. As in [1], we may assume there is a horoball tangent to the horoball at $\infty$ centered at 0 . Furthermore, the cusp shape and the cusp volumes provide a peripheral translation of length $\sqrt{3}$. Thus there is also a full sized horoball centered at $\sqrt{3}$. We claim that such a diagram will look like Figure 9. First, we will explain the labeling. In this figure, $O=0, Y=(\sqrt{3}+i) / 2$, $X=(\sqrt{3}-i) / 2$ and $D=\sqrt{3}$ and there are horoballs that are tangent to the horoball at $\infty$, which are of Euclidean diameter 1 tangent to $\partial \mathbb{H}^{3}$ at 0 and $D$. Also, there are elliptic elements of order 3 in $\Gamma_{Q}$ fixing 0 and $\infty, X$ and $\infty, Y$ and $\infty$, and $D$ and $\infty$.

By Proposition 5.3 the point stabilizer of $0+j$ is $D_{3}$ or $C_{3}$. 


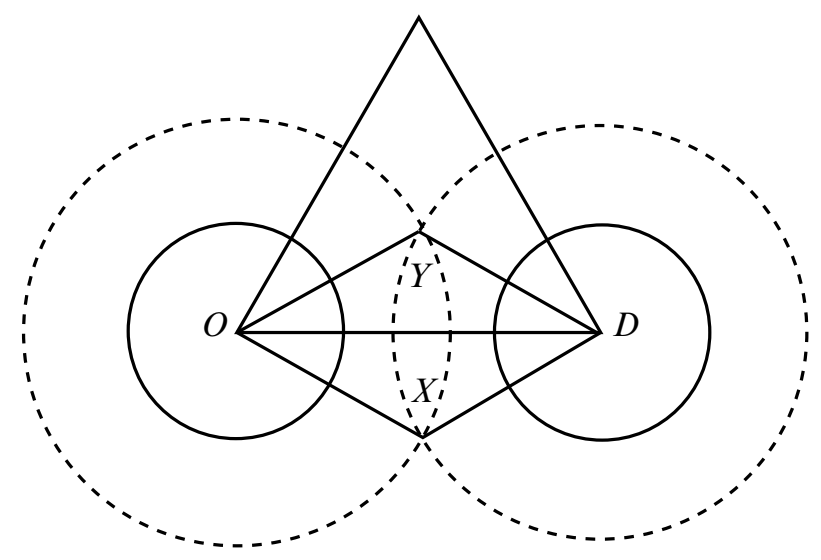

Figure 9: A fundamental domain for $Q$ lies above $O X D Y$

Case 1 Assume the point stabilizer of $0+j$ is $D_{3}$.

Then there is an element $\gamma=\left(\begin{array}{cc}0 & i e^{i \theta} \\ i e^{-i \theta} & 0\end{array}\right)$ that fixes $e^{i \theta}$ and $-e^{i \theta}$. Therefore, the isometric sphere corresponding to $\gamma$ has radius 1 and is centered at 0 . Also, let $r$ be the element of order 3 fixing $Y$ and $\infty$. Then

$$
r=\left(\begin{array}{cc}
\omega & (\sqrt{3}+i) / 2 \\
0 & \omega^{-1}
\end{array}\right)
$$

and $r \gamma r^{-1}$ admits an isometric sphere of radius 1 centered at $\sqrt{3}$. The boundaries of these isometric spheres in $\mathbb{C}$ are depicted by dotted lines in Figure 9. Finally, let $t=\left(\begin{array}{cc}1 & \sqrt{3} \\ 0 & 1\end{array}\right)$.

Since $\Gamma^{\prime}=\langle\gamma, r, t\rangle$ is a subgroup with finite covolume (see Figure 9), it must be of finite index in $\Gamma_{Q}$. Also, by combining the assumption that cusp volume is $\sqrt{3} / 4$ with the upper bound on the cusp density of $2 /\left(v_{0} \sqrt{3}\right)$ (see Section 2.1), we know that covolume $\left(\Gamma_{Q}\right) \geq v_{0} / 2$. Hence $\left[\Gamma_{Q}: \Gamma^{\prime}\right]=1,2$.

If $\left[\Gamma_{Q}: \Gamma^{\prime}\right]=2$, then covolume $\left(\Gamma_{Q}\right)=v_{0} / 2$ and there are horoballs based at $(\sqrt{3}+i) / 2$ and $(\sqrt{3}-i) / 2$ of Euclidean diameter 1 . Thus, by Proposition 5.3, the point stabilizers above these points are either both $D_{3}$ or both $C_{3}$. Hence the cusp corresponds to a vertex in the isotropy graph that is either connected to three vertices labeled by $D_{3}$ isotropy groups, or there is a loop labeled by 3-torsion and the cusp connects to one vertex labeled by a $D_{3}$ isotropy group (see Figure 10 ). In either case, $\Gamma_{Q}$ cannot be trivial under the cusp-killing homomorphism.

Therefore, we consider the case that $\left[\Gamma_{Q}: \Gamma^{\prime}\right]=1$. Here, $\operatorname{tr}(\gamma \cdot r)=-i((\sqrt{3}+i) / 2) e^{-i \theta}$. Since $-i(\sqrt{3}+i) / 2$ is a unit and we are assuming integral traces, $e^{-i \theta}$ is an algebraic 

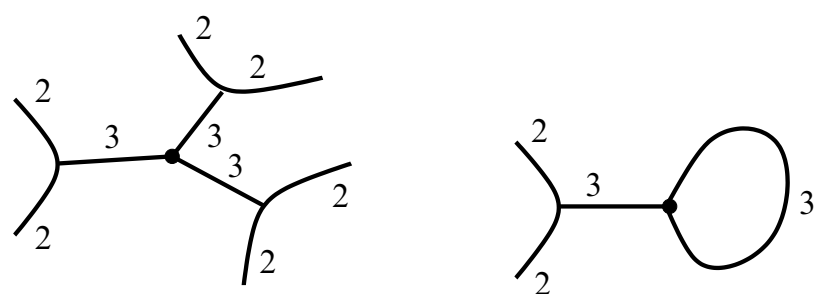

Figure 10: The two possible types of isotropy graphs for the orbifold $Q$ described in Case 1 of Lemma 5.4

integer. Hence

$$
\left\langle\left(\begin{array}{cc}
0 & i e^{i \theta} \\
i e^{-i \theta} & 0
\end{array}\right),\left(\begin{array}{cc}
1 & \sqrt{3} \\
0 & 1
\end{array}\right),\left(\begin{array}{cc}
\omega & \frac{\sqrt{3}+i}{2} \\
0 & \omega^{-1}
\end{array}\right)\right\rangle
$$

is a representation of $\Gamma_{Q}$ with parabolic elements fixing 0 and $\infty$ where all entries of the generators are algebraic integers. If $\Gamma_{K} \subset \Gamma_{Q}$, then $\Gamma_{K}$ admits an integral representation. However, the maximal abelian subgroup $A_{Q}$ of $P_{Q}$ is of the form

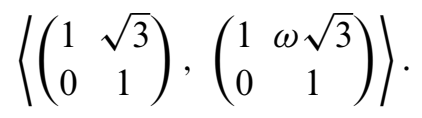

In particular, $A_{Q}$ vanishes under reduction modulo the prime ideal $I$, if $\sqrt{3} \in I$. Therefore, no knot group $\Gamma_{K}$ is a subgroup of $\Gamma_{Q}$ by Lemma 3.2.

Case 2: The point stabilizer of $0+\boldsymbol{j}$ is $\boldsymbol{C}_{\mathbf{3}}$ In this case, there is a group element $\gamma^{\prime}$ that identifies $0+j$ with a point above either $(\sqrt{3}+i) / 2$ or $(\sqrt{3}-i) / 2$. We may assume that $0+j$ is identified with $(\sqrt{3}+i) / 2+c j$. Since $\gamma^{\prime}$ can be decomposed into reflections in the plane defined by hemisphere of radius 1 centered at 0 and vertical planes, $0+j$ and $(\sqrt{3}+i) / 2+c j$ are the same Euclidean distance above $\mathbb{C}$ and $c=1$.

Hence, under $\gamma^{\prime}, \infty \mapsto 0,(\sqrt{3}+i) / 2 \mapsto \infty$ and $(\sqrt{3}+i) / 2+j \mapsto j$. Here

$$
\gamma^{\prime}=\left(\begin{array}{cc}
0 & (\sqrt{3}+i) / 2 \\
(-\sqrt{3}+i) / 2 & 1
\end{array}\right) .
$$

Let

$$
r^{\prime}=\left(\begin{array}{cc}
\omega & (-\sqrt{3}-3 i) / 2 \\
0 & \omega^{-1}
\end{array}\right)
$$

Since $\gamma^{\prime}$ admits an isometric sphere of radius 1 at $(\sqrt{3}+i) / 2$, then $\gamma^{\prime-1}$ admits an isometric sphere of radius 1 at 0 , and $r^{\prime} \cdot \gamma^{\prime-1} \cdot r^{\prime-1}$ admits an isometric sphere of radius 1 at $\sqrt{3}$. 
Hence by $\Gamma_{3}=\left\langle\gamma^{\prime}, r^{\prime}, t\right\rangle$ is a subgroup of finite covolume ( $t$ is defined in Case 1$)$ and $\Gamma_{3}$ is of finite index in $\Gamma_{Q}$. In this case, $k \Gamma_{3}=\mathbb{Q}(\sqrt{-3})$ and $\Gamma_{3}$ has integral traces. Thus, by [10, Theorem 8.3.2], $\Gamma_{3}$ is arithmetic and therefore $\Gamma_{Q}$ is arithmetic. Again by [21], the only knot complement that can cover $Q$ is the figure- 8 knot complement. Cusp volume considerations would force the figure- 8 knot complement to be a 4 -fold cover of $Q$. However, $Q$ has 3-torsion on the cusp. Hence in this case, $Q$ is not covered by a hyperbolic knot complement.

Finally, if $Q$ has a $S^{2}(2,3,6)$ cusp and cusp volume $\sqrt{3} / 8$ and $\Gamma_{Q}$ admits integral traces, then the point stabilizer of $0+j$ is $D_{6}$. Hence an identical argument to Case 1 shows $Q$ is not covered by a knot complement.

We now expand upon Lemma 4.3. However, unlike that lemma, we assume that $p: M \rightarrow Q$, where $M$ is a manifold.

Lemma 5.5 Let $p: M \rightarrow Q$, where $M$ is a manifold covered by a hyperbolic knot complement.

(1) If $Q$ has a $S^{2}(3,3,3)$ cusp, then $\operatorname{deg}(p)=12 n n \geq 1$.

(2) If $Q$ has a $S^{2}(2,4,4)$ cusp, then the $\operatorname{deg}(p) \geq 24$.

Proof (1) Assume that $Q$ has a $S^{2}(3,3,3)$ cusp. First, consider the isotropy graph of $Q$. If there is a loop in the isotropy graph, then the other edge emanating from the cusp cannot connect the cusp to a point with isotropy group $D_{3}$. In this case, $\Gamma_{Q}$ would be nontrivial under the cusp-killing homomorphism (see Figure 11).

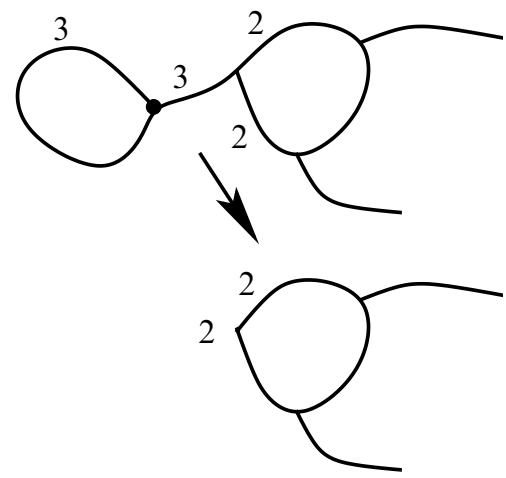

Figure 11: Application of the cusp-killing homomorphism to a graph with a cycle labeled 3 and a vertex labeled $D_{3}$

Therefore this vertex is fixed by a group $G$, where $G$ is either $A_{4}, S_{4}$ or $A_{5}$. For $G$ to lift to a torsion-free group, $\operatorname{deg}(p)$ must be a multiple of the order of $G$. Hence $\operatorname{deg}(p)=12 n(n \in \mathbb{Z})$. 
If we assume that there is no edge in the isotropy graph with both endpoints on the cusp, then there must be at least one vertex adjacent to the cusp labeled with a $A_{4}, S_{4}$ or $A_{5}$ subgroup. Otherwise, all vertices are labeled with $D_{3}$ and just as above, $\Gamma_{Q}$ would be nontrivial under the cusp-killing homomorphism. Thus $\operatorname{deg}(p)=12 n$.

(2) Assume that $Q$ has a $S^{2}(2,4,4)$ cusp. Since $S^{3}-K$ is a manifold, all isotropy subgroups of $\Gamma_{Q}$ must vanish in the lift. Either the order 4 elements in the cusp are part of the same fixed axis (see Figure 4) or the 4-torsion on the cusp connects to a pair of distinct vertices in the isotropy graph.

In the first case, $\operatorname{deg}(p) \geq 24$ by Proposition 4.2. In the second case, the vertices are either of type $D_{4}$ or $S_{4}$ isotropy subgroup. If there is a vertex of type $S_{4}$, then $\operatorname{deg}(p) \geq 24$. If we have a vertex of type $D_{4}$, there must be some edges in the isotropy graph labeled with odd integers otherwise the graph would be nontrivial under the cusp-killing homomorphism. Since $M$ is a manifold, $\Gamma_{M}$ is torsion free. Thus $\operatorname{deg}(p)=8(2 k+1) n$ for some $n, k \geq 1$ and $\operatorname{deg}(p) \geq 24$.

\subsection{Length, area and volume on the cusp}

We discuss length for peripheral elements fixing $\infty$ below. Let $Q$ be a 1-cusped hyperbolic 3-orbifold and fix a representation for $\Gamma_{Q}$ in $\operatorname{PSL}(2, \mathbb{C})$ such that $P_{Q}$ is upper triangular and we consider $\Gamma_{Q}$ acting on upper half space. Denote by $1 / c$ the height of a maximal horoball tangent to $\infty$ and denote by $S_{c}$ the horosphere centered at $\infty$ of Euclidean height $1 / c$. If $\gamma \in \Gamma_{Q}$ is a parabolic element fixing $\infty$, we measure $\operatorname{len}(\gamma)$ by its translation length in $S_{c}$. If $\gamma=\left(\begin{array}{ll}1 & x \\ 0 & 1\end{array}\right)$, then $\operatorname{len}(\gamma)=c \cdot|x|$. Therefore, if $\gamma$ corresponds to an exceptional slope, then by the six theorem $c \cdot|x| \leq 6$ (see [2; 9]). Finally, by Lemma 3.2, we will only consider representations of $\Gamma_{Q}$ such that $\left(\begin{array}{ll}1 & 1 \\ 0 & 1\end{array}\right) \in \Gamma_{Q}$. Since the interiors of maximal horoballs are disjoint, we know that $c \geq 1$. Additionally, $\left(\begin{array}{ll}1 & x \\ 0 & 1\end{array}\right)$ and $\left(\begin{array}{cc}1 & -x \\ 0 & 1\end{array}\right)$ correspond to the same slopes in terms of Dehn surgery parameters, so for convenience we consider them as one curve in our accounting of short parabolic elements.

With $\Gamma_{Q}, P_{Q}$ as above, recall that if $A$ is the area of the fundamental domain for $P_{Q}$ in the horosphere of Euclidean height 1, the cusp volume of $Q$ is

$$
\int_{\frac{1}{c}}^{\infty} \frac{A}{z} d z=\frac{c^{2} \cdot A}{2} .
$$

Proof of Theorem 1.2 Assume $M$ admits two exceptional surgeries, $M$ is not covered by the figure- 8 knot complement, and $M$ is covered by a small knot complement. By [21], the second assumption is equivalent to $M$ being nonarithmetic and by [4], the third hypothesis implies that $\Gamma_{M}$ has integral traces. Also, assume $p: M \rightarrow Q$, 
where $Q$ has a rigid cusp (see Section 2.1). Finally, using Lemma 3.1, we assume that $\Gamma_{Q} \subset \operatorname{PSL}(2, \mathbb{A})$ and $P_{Q}$ is upper triangular. We break the proof into three cases, one for each cusp type of $Q$.

Case 1: $\boldsymbol{Q}$ has a $\boldsymbol{S}^{\mathbf{2}}(\mathbf{2}, \mathbf{4}, \mathbf{4})$ cusp By Lemma 3.2, $\left(\begin{array}{ll}1 & 1 \\ 0 & 1\end{array}\right) \in P_{M}$. By the six theorem (see [2; 9]), $P_{M}$ must have another short peripheral curve. Combining the bound $\operatorname{deg}(p) \geq 24$ from Lemma 5.5 with $P_{Q} \subset \operatorname{PSL}(2, \mathbb{A})$ and Lemma 4.4,

$$
P_{M}=\left\langle\left(\begin{array}{ll}
1 & 1 \\
0 & 1
\end{array}\right),\left(\begin{array}{cc}
1 & 6 i \\
0 & 1
\end{array}\right)\right\rangle
$$

and $c=1$ (with $c$ defined above). In this case, $\operatorname{deg}(p)=24$. Hence (1) shows the cusp volume of $M$ is 3 and therefore the cusp volume of $Q$ is $\frac{3}{24}$. There is one such orbifold with cusp volume $\frac{3}{24}$, which is arithmetic, contradicting our hypothesis.

Case 2: $\boldsymbol{Q}$ has a $\boldsymbol{S}^{\mathbf{2}}(\mathbf{3}, \mathbf{3}, \mathbf{3})$ cusp By Lemmas 3.2 and 4.4 , we can find a representation for $\Gamma_{M}$ where

$$
P_{M}=\left\langle\left(\begin{array}{ll}
1 & 1 \\
0 & 1
\end{array}\right),\left(\begin{array}{cc}
1 & n \omega \\
0 & 1
\end{array}\right)\right\rangle
$$

such that $\omega^{2}+\omega+1=0$. By the six theorem (see [2;9]), $n \leq 6$. However, $3 n$ must be a multiple of 12 . Hence, by Lemma $5.5, \operatorname{deg}(p)=12$ and so $n=4$. Here the two shortest parabolic elements (excluding inverses) are $\mu=\left(\begin{array}{ll}1 & 1 \\ 0 & 1\end{array}\right)$ and $\lambda=\left(\begin{array}{ccc}1 & 2+4 \omega \\ 0 & 1\end{array}\right)$ $(|2+4 \omega|=2 \sqrt{3})$.

In order to have two curves $\gamma_{1}, \gamma_{2} \in P_{M}$ with $\operatorname{len}\left(\gamma_{i}\right) \leq 6$, the horoballs tangent to $B_{\infty}$ have Euclidean height greater than $1 / \sqrt{3}$ and so $c \leq \sqrt{3}$. (Note if $\lambda$ is a longer element, say $\lambda=\left(\begin{array}{cc}1 & 3+4 \omega \\ 0 & 1\end{array}\right)$, then we must have $\left.c \leq 6 / \sqrt{13}<\sqrt{3}\right)$. Thus the cusp volume of $M$ is in the range $[\sqrt{3}, 3 \sqrt{3}](1 \leq|c| \leq \sqrt{3})$ and the cusp volume of $Q$ is in the range $[\sqrt{3} / 12, \sqrt{3} / 4]$.

Since any orbifold $Q$ with $S^{2}(3,3,3)$ cusp and cusp volume $\sqrt{3} / 12, \sqrt{3} / 6$ or $1 / 4$ is arithmetic (see Proposition 2.2), these orbifolds are excluded by hypothesis. By Theorem 2.1, the only possible cusp volumes for $Q$ are $(3 \sqrt{3}+\sqrt{15}) / 24, \sqrt{21} / 12$ or $\sqrt{3} / 4$. The first case implies that $Q$ is $\mathbb{H}^{3} / \Gamma(5,2,2,3,3,3)$ which has an order 60 isotropy group fixing a point (see [10, Section 4.7]). Thus it cannot have a 12-fold manifold cover. In the second case, we know no such orbifold can be covered by a knot complement by Lemma 5.2. Finally, we show the third case cannot occur by appealing to Lemma 5.4 .

Case 3: $Q$ has $S^{2}(2,3,6)$ and $M$ admits a nontrivial symmetry Since the element of 6 torsion is part of a dihedral group of order 12 , we know $\operatorname{deg}(p)=12 n$. 
If $\operatorname{deg}(p)>24$, we claim $M$ cannot admit two exceptional surgeries. In this case, again by Lemmas 3.2 and 4.4,

$$
P_{M}=\left\langle\left(\begin{array}{ll}
1 & 1 \\
0 & 1
\end{array}\right),\left(\begin{array}{cc}
1 & 2 n \omega \\
0 & 1
\end{array}\right)\right\rangle
$$

with $n>3$ and $\omega^{2}+\omega+1=0$. Since $|c| \geq 1$, if $\gamma \in P_{M}$ with $\operatorname{len}(\gamma) \leq 6$, then $\gamma=\left(\begin{array}{cc}1 & \pm 1 \\ 0 & 1\end{array}\right)$. However, these curves both correspond to surgery along the meridian.

If $\operatorname{deg}(p)=24$, then $P_{M}$ is same as in Case 2 above. Therefore $M$ has cusp volume in $[\sqrt{3}, 3 \sqrt{3}]$ and $Q$ has cusp volume in $[\sqrt{3} / 24, \sqrt{3} / 8]$. For cusp volume in $[\sqrt{3} / 24, \sqrt{3} / 8)$, these orbifolds fit Adams' list and only the figure- 8 knot complement can 24-fold cover $Q$. This follows from Case 2 above. If the cusp volume is exactly $\sqrt{3} / 8$, we appeal to Lemma 5.4.

If $\operatorname{deg}(p)=12$, we may consider $\Gamma_{Q}=P_{Q} \cdot \Gamma_{M}$. In this case,

$$
P_{Q}=\left\langle r=\left(\begin{array}{cc}
\ell & 0 \\
0 & \ell^{-1}
\end{array}\right), t=\left(\begin{array}{ll}
1 & \omega \\
0 & 1
\end{array}\right), \mu=\left(\begin{array}{ll}
1 & 1 \\
0 & 1
\end{array}\right)\right\rangle,
$$

where $\ell=e^{i \pi / 6}$ and $\mu$ is the meridian of the knot complement which covers $M$.

Since $t \notin \Gamma_{M}$, we first note that $r, r^{2} \notin N\left(\Gamma_{M}\right)$, where $N\left(\Gamma_{M}\right)$ is the normalizer of $\Gamma_{M}$ in PSL(2, $\left.\mathbb{C}\right)$. Also, $t \notin N\left(\Gamma_{M}\right)$ by Lemma 4.3.

Therefore the only symmetry $M$ may admit can be realized by $r^{3}$ and we may assume $N\left(\Gamma_{M}\right)=\left\langle r^{3}, \Gamma_{M}\right\rangle$. Then the conjugates of $\Gamma_{M}$ in $P_{Q} \cdot \Gamma_{M}$ are $\Gamma_{M}, r \cdot \Gamma_{M} \cdot r^{-1}$, $r^{2} \cdot \Gamma_{M} \cdot r^{-2}, t \cdot \Gamma_{M} \cdot t^{-1}, r t \cdot \Gamma_{M} \cdot(r t)^{-1}$ and $r^{2} t \cdot \Gamma_{M} \cdot t^{-1} r^{-2}$. In this case, $t$ maps to a product of three $2-$ cycles in $S_{6}$. Hence $P_{Q} \cdot \Gamma_{M}$ has a $\mathbb{Z} / 2 \mathbb{Z}$ quotient. Therefore $Q$ is covered by an orbifold $Q^{\prime}$ with a $S^{2}(3,3,3)$ cusp with $\left[P_{Q}: P_{Q^{\prime}}\right]=2$ and $\Gamma_{Q^{\prime}}=P_{Q^{\prime}} \cdot \Gamma_{M}$ by Proposition 4.1. However $M$ would be a 6 -fold cover of $Q^{\prime}$, where $Q^{\prime}$ has a $S^{2}(3,3,3)$ cusp. This is a contraction to the minimum degree cover of $p^{\prime}: M \rightarrow Q^{\prime}$ established by Lemma 5.5.

\section{Further remarks}

We conclude by noting that there are knot complements that admit exceptional surgeries and are not small. In fact, by work of Baker (see [3]), there are knot complements that admit (nontrivial) finite cyclic fillings that are not small. It remains unknown whether any of these knot complements admit hidden symmetries.

In the case where the knot complements cover a manifold of small volume, more can be said. Let us consider manifolds $\beta_{n, m}$ that arise from $(n, m)$ surgery on one cusp 
of the Berge manifold as in [7]. That paper shows the existence of an infinite family of commensurability classes such that each commensurability class contains exactly three knot complements and a $\beta_{n, m}$. However, the argument involves a geometric limit of groups so finitely many commensurability classes in this family may admit hidden symmetries. The following theorem provides a strengthening of the main theorem of [7] in the sense that the argument produces an explicit family of commensurability classes containing exactly three knot complements.

First, however, we must exclude the 6 slopes that correspond to nonhyperbolic fillings of the Berge manifold (see Martelli and Petronio [12, Remark A.3, Table 7]). In that paper, the authors refer to a census manifold description of the Berge manifold, which has now been updated. The manifold in question was called $M 4_{3}^{2}$, later called $m 202(\cdot),(\cdot)$, and is currently the $150^{\text {th }}$ manifold in SNAPPY's OrientableCuspedCensus (see the documentation for [6] for further background). Using SNAPPY's standard framing on the $150^{\text {th }}$ manifold in the OrientableCuspedCensus, these slopes form the set

$$
S=\{(1,0),(0,1),(-1,1),(1,1),(-2,1),(-1,2)\} .
$$

We note that both cusps are framed such that $S$ is the set of exceptional slopes.

Theorem 6.1 If $(n, 7)=1,(n, m)=1$ and $(n, m) \notin S$, then the commensurability class of the manifold arising from $(n, m)$ surgery on one cusp of the Berge manifold contains exactly three knot complements.

Proof Fix a pair $(n, m)$ with $(n, 7)=1,(n, m)=1$ and $(n, m) \notin S$. Then $\beta_{n, m}$ is covered by three hyperbolic knot complements since $(n, 7)=1$ (see [7, Lemma 3.1]). Also, $\beta_{n, m}$ is a manifold since $(n, m)=1$. Therefore, if $\beta_{n, m}$ does not cover a rigid cusped orbifold, then there are exactly three knot complements in the commensurability class of $\beta_{n, m}$ (see [5, Theorem 1.2]). The remainder of the proof will be dedicated to showing $\beta_{n, m}$ does not cover a rigid cusped orbifold.

We note that the volume of the Berge manifold is $4 v_{0}$, where just as before $v_{0}$ is the volume of a the regular ideal tetrahedron. Hence $\operatorname{vol}\left(\beta_{n, m}\right)<4 v_{0}$ by Thurston's hyperbolic Dehn surgery theorem [24, Theorem 5.8.2].

Suppose $Q$ is an orbifold with a $S^{2}(3,3,3)$ or $S^{2}(2,4,4)$ cusp that is covered by $\beta_{n, m}$. By Lemma 5.5, $\operatorname{vol}(Q)<v_{0} / 3$ in the $S^{2}(3,3,3)$ case and $\operatorname{vol}(Q)<v_{0} / 6$ in the $S^{2}(2,4,4)$ case. In either case, by Proposition 2.2 , such a $Q$ would be arithmetic and therefore could not be covered by more than one knot complement by [21].

If $Q$ has a $S^{2}(2,3,6)$ cusp, then we use the fact that $Q$ must have an isotropy group which is dihedral and order 12 . Hence, if $p: \beta_{n, m} \rightarrow Q, \operatorname{deg}(p)=12 n$. If 
$\operatorname{deg}(p) \geq 24$, then $\operatorname{vol}(Q)<v_{0} / 6$ and would be arithmetic by Proposition 2.2 and just as above, this is a contradiction to [21] as these orbifolds are covered by more than one knot complement. If $\operatorname{deg}(p)=12$, we note that since the Berge manifold is strongly invertible, $\beta_{n, m}$ can be realized as the double branched cover of a tangle filling on the quotient of the Berge manifold under a strong inversion. Hence $\beta_{n, m}$ admits a symmetry. Therefore, we may appeal to the proof of Theorem 1.2 to see that there can be no such covering map $p$ of degree 12 . Therefore, no manifold $\beta_{n, m}$ can cover an orbifold with a rigid cusp.

\section{References}

[1] C C Adams, Noncompact hyperbolic 3-orbifolds of small volume, from: "Topology '90', (B Apanasov, W D Neumann, A W Reid, L Siebenmann, editors), Ohio State Univ. Math. Res. Inst. Publ. 1, de Gruyter, Berlin (1992) 1-15 MR1184398

[2] I Agol, Bounds on exceptional Dehn filling, Geom. Topol. 4 (2000) 431-449 MR1799796

[3] K Baker, Closed essential surfaces in the complements of large volume Berge knots Available at http://front.math.ucdavis.edu/math.GT/0509082

[4] H Bass, Groups of integral representation type, Pacific J. Math. 86 (1980) 15-51 MR586867

[5] M Boileau, S Boyer, R Cebanu, G S Walsh, Knot commensurability and the Berge conjecture, Geom. Topol. 16 (2012) 625-664 MR2928979

[6] M Culler, N Dunfield, J Weeks, Snappy: A computer program for studying the geometry and topology of 3-manifolds Available at http://snappy. computop.org

[7] N Hoffman, Commensurability classes containing three knot complements, Algebr. Geom. Topol. 10 (2010) 663-677 MR2606796

[8] H Koch, Number theory, Graduate Studies in Mathematics 24, Amer. Math. Soc. (2000) MR1760632

[9] M Lackenby, Word hyperbolic Dehn surgery, Invent. Math. 140 (2000) 243-282 MR1756996

[10] C Maclachlan, A W Reid, The arithmetic of hyperbolic 3-manifolds, Graduate Texts in Mathematics 219, Springer (2003) MR1937957

[11] G A Margulis, Discrete subgroups of semisimple Lie groups, Ergeb. Math. Grenzgeb. 17, Springer, Berlin (1991) MR1090825

[12] B Martelli, C Petronio, Dehn filling of the "magic" 3-manifold, Comm. Anal. Geom. 14 (2006) 969-1026 MR2287152

[13] R Meyerhoff, The cusped hyperbolic 3-orbifold of minimum volume, Bull. Amer. Math. Soc. 13 (1985) 154-156 MR799800 
[14] J W Morgan, The Smith conjecture, from: "The Smith conjecture", (J W Morgan, H Bass, editors), Pure Appl. Math. 112, Academic Press, Orlando, FL (1984) 3-6 MR758460

[15] J Morgan, G Tian, Ricci flow and the Poincaré conjecture, Clay Mathematics Monographs 3, Amer. Math. Soc. (2007) MR2334563

[16] G D Mostow, Quasiconformal mappings in n-space and the rigidity of hyperbolic space forms, Inst. Hautes Études Sci. Publ. Math. (1968) 53-104 MR0236383

[17] W D Neumann, A W Reid, Arithmetic of hyperbolic manifolds, from: “Topology '90", (B Apanasov, W D Neumann, A W Reid, L Siebenmann, editors), Ohio State Univ. Math. Res. Inst. Publ. 1, de Gruyter, Berlin (1992) 273-310 MR1184416

[18] W D Neumann, A W Reid, Notes on Adams' small volume orbifolds, from: “Topology '90', (B Apanasov, W D Neumann, A W Reid, L Siebenmann, editors), Ohio State Univ. Math. Res. Inst. Publ. 1, de Gruyter, Berlin (1992) 311-314 MR1184417

[19] G Prasad, Strong rigidity of Q-rank 1 lattices, Invent. Math. 21 (1973) 255-286 MR0385005

[20] A W Reid, A note on trace-fields of Kleinian groups, Bull. London Math. Soc. 22 (1990) 349-352 MR1058310

[21] A W Reid, Arithmeticity of knot complements, J. London Math. Soc. 43 (1991) 171-184 MR1099096

[22] A W Reid, GS Walsh, Commensurability classes of 2-bridge knot complements, Algebr. Geom. Topol. 8 (2008) 1031-1057 MR2443107

[23] R K W Roeder, Compact hyperbolic tetrahedra with nonobtuse dihedral angles, Publ. Mat. 50 (2006) 211-227 MR2325019

[24] W P Thurston, The geometry and topology of 3-manifolds, Princeton Univ. Math. Dept. Lecture Notes (1979) Available at http://msri.org/publications/books/ gt $3 \mathrm{~m} /$

[25] G S Walsh, Orbifolds and commensurability, from: "Interactions between hyperbolic geometry, quantum topology and number theory", (A Champanerkar, O Dasbach, E Kalfagianni, I Kofman, W Neumann, N Stoltzfus, editors), Contemp. Math. 541, Amer. Math. Soc. (2011) 221-231 MR2796635

Department of Mathematics and Statistics, University of Melbourne Parkville, Victoria 3010, Australia nhoffman@ms . unimelb.edu.au

Received: 15 December 2011 Revised: 9 December 2013 